\title{
The Gaia-ESO Survey: Reevaluation of the parameters of the open cluster Trumpler 20 using photometry and spectroscopy $\star, \star \star$
}

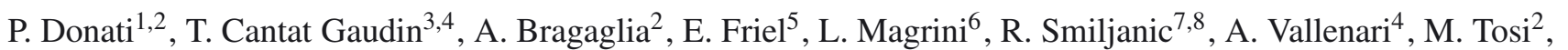

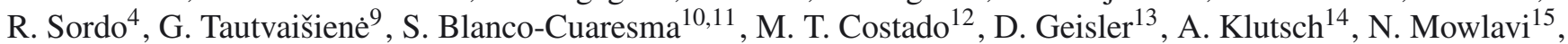
C. Muñoz ${ }^{13}$, I. San Roman ${ }^{13}$, S. Zaggia ${ }^{4}$, G. Gilmore ${ }^{16}$, S. Randich ${ }^{6}$, T. Bensby ${ }^{17}$, E. Flaccomio ${ }^{18}$, S. E. Koposov ${ }^{16,19}$, A. J. Korn ${ }^{20}$, E. Pancino ${ }^{2,21}$, A. Recio-Blanco ${ }^{22}$, E. Franciosini ${ }^{6}$, P. de Laverny ${ }^{22}$, J. Lewis ${ }^{16}$, L. Morbidelli ${ }^{6}$,

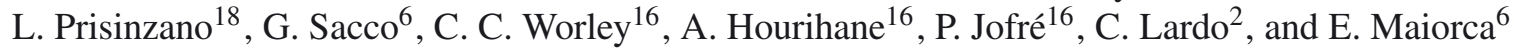

1 Dipartimento di Fisica e Astronomia, Università di Bologna, via Ranzani 1, 40127 Bologna, Italy e-mail: paolo.donati4@unibo.it

2 INAF - Osservatorio Astronomico di Bologna, via Ranzani 1, 40127 Bologna, Italy

3 Dipartimento di Fisica e Astronomia, Università di Padova, vicolo Osservatorio 3, 35122 Padova, Italy

4 INAF - Osservatorio Astronomico di Padova, vicolo Osservatorio 5, 35122 Padova, Italy

5 Department of Astronomy, Indiana University, Bloomington, IN 47405, USA

${ }^{6}$ INAF - Osservatorio Astrofisico di Arcetri, Largo E. Fermi, 5, 50125 Firenze, Italy

7 European Southern Observatory, Karl-Schwarzschild-Str. 2, 85748 Garching bei München, Germany

8 Department for Astrophysics, Nicolaus Copernicus Astronomical Center, ul. Rabiańska 8, 87-100 Toruń, Poland

9 Institute of Theoretical Physics and Astronomy, Vilnius University, Goštauto 12, 01108 Vilnius, Lithuania

10 Univ. Bordeaux, LAB, UMR 5804, 33270 Floirac, France

11 CNRS, LAB, UMR 5804, 33270 Floirac, France

12 Instituto de Astrofísica de Andalucía, CSIC, Apdo 3004, 18080 Granada, Spain

13 Departamento de Astronomía, Universidad de Concepción, 160-C Casilla, Concepción, Chile

14 INAF - Osservatorio Astrofisico di Catania, via S. Sofia 78, 95123 Catania, Italy

15 Astronomy Department, University of Geneva, 1211 Geneva, Switzerland

16 Institute of Astronomy, University of Cambridge, Madingley Road, Cambridge CB3 0HA, UK

17 Lund Observatory, Department of Astronomy and Theoretical Physics, Box 43, 22100 Lund, Sweden

18 INAF - Osservatorio Astronomico di Palermo, Piazza del Parlamento 1, 90134 Palermo, Italy

19 Moscow M.V. Lomonosov State University, Sternberg Astronomical Institute, Universitetskij pr., 13, 119992 Moscow, Russia

20 Department of Physics and Astronomy, Uppsala University, Box 516, 75120 Uppsala, Sweden

21 ASI Science Data Center, via del Politecnico SNC, 00133 Roma, Italy

22 Laboratoire Lagrange (UMR7293), Université de Nice Sophia Antipolis, CNRS, Observatoire de la Côte d'Azur, BP 4229, 06304 Nice Cedex 4, France

Received 25 October 2013 / Accepted 5 December 2013

\section{ABSTRACT}

Trumpler 20 is an old open cluster (OC) located toward the Galactic centre, at about $3 \mathrm{kpc}$ from the Sun and $7 \mathrm{kpc}$ from the Galactic centre. Its position makes this cluster particularly interesting in the framework of the chemical properties of the Galactic disc because very few old OCs reside in the inner part of the disc. For this reason it has been selected as a cluster target of the Gaia-ESO Survey, and spectra of many stars in the main-sequence and red-clump phases are now available. Moreover, although it has been studied by several authors in the past, no consensus on the evolutionary status of $\operatorname{Tr} 20$ has been reached. The heavy contamination of field stars (the line of sight of $\operatorname{Tr} 20$ crosses the Carina spiral arm) complicates a correct interpretation. Another interesting aspect of the cluster is that it shows a broadened main-sequence turn-off and a prominent and extended red-clump, characteristics that are not easily explained by classical evolutionary models. Exploiting both spectroscopic information from the Gaia-ESO Survey (and the ESO archive) and literature photometry, we obtain a detailed and accurate analysis of the properties of the cluster. We make use of the first accurate metallicity measurement ever obtained from several spectra of red clump stars, and of cluster membership determination using radial velocities. According to the evolutionary models adopted, we find that $\operatorname{Tr} 20$ has an age in the range $1.35-1.66 \mathrm{Gyr}$, an average reddening $E(B-V)$ in the range $0.31-0.35 \mathrm{mag}$, and a distance modulus $(m-M)_{0}$ between 12.64 and 12.72 mag. The spectroscopic metallicity is $[\mathrm{Fe} / \mathrm{H}]=+0.17 \mathrm{dex}$. We discuss the structural properties of the object and constrain possible hypotheses for its broadened upper main sequence by estimating the effect of differential reddening and its extended red clump.

Key words. open clusters and associations: general - Hertzsprung-Russell and C-M diagrams

^ Based on the data obtained at ESO telescopes under programme 188.B-3002 (the public Gaia-ESO spectroscopic survey, PIs Gilmore \& Randich) and on the archive data of the programme 083.D-0671.

$\star \star$ Full Table 3 and the photometric catalogue with differential reddening corrections are only available at the CDS via anonymous ftp to cdsarc.u-strasbg.fr (130.79.128.5) or via http://cdsarc.u-strasbg.fr/viz-bin/qcat? J/A+A/561/A94 


\section{Introduction}

The Gaia-ESO Survey (GES, see Gilmore et al. 2012; Randich \& Gilmore 2013) is a large, public spectroscopic survey of the Galaxy using the high-resolution multi-object spectrograph FLAMES (see Pasquini et al. 2002) on the Very Large Telescope (ESO, Chile). It targets about $10^{5}$ stars and covers the bulge, thick and thin discs, and halo components, as well as a sample of about 100 open clusters (OCs) of all ages, metallicities, locations, and masses. While the Gaia-ESO Survey will leave an unprecedented legacy for high-resolution spectroscopic observations, its value can even be increased by synergies with other missions. In the long run, the Gaia satellite will produce distances and proper motions for all objects targeted during the Gaia-ESO Survey, which has in fact been conceived also as a ground-based complement to Gaia. On shorter timescales, information present in the archives and literature plays a fundamental role in enhancing both the efficiency of the spectroscopic observations and the scientific return of the survey. For instance, photometry of the Gaia-ESO Survey targets is essential for a full understanding of their physical parameters. In this framework we present a comprehensive and homogeneous analysis of the archive photometry and the Gaia-ESO Survey spectroscopy of one of the Gaia-ESO Survey OC targets: Trumpler 20.

The open cluster Trumpler $20(\operatorname{Tr} 20)$ is a relatively old OC located in the fourth quadrant of the Galactic plane $(\mathrm{RA}=12: 39: 32$, Dec $=-60: 37: 36$, Seleznev et al. 2010; $l=301.475^{\circ}, b=2.221^{\circ}$, Dias et al. 2002) as shown in Fig. 1. Only a few studies are available (see Sect. 2). There is no consensus on the evolutionary status of $\operatorname{Tr} 20$ : its position in the Galactic disc is such that many field interlopers pollute the main evolutionary phases on the colour-magnitude diagram (CMD), jeopardising the derivation of the cluster parameters. Moreover, the reddening, and in particular the differential reddening (DR) across the face of the cluster, plays a considerable role in shaping the CMD morphology (see e.g., Platais et al. 2008, 2012). $\operatorname{Tr} 20$ shows a peculiar morphology of the red-clump (RC) phase, common to other OCs (see e.g., Mermilliod \& Mayor 1989; Mermilliod et al. 1998; Girardi et al. 2000b), but still little explained and hardly understood (see Sect. 4.3 for a discussion). Finally, prior to the Gaia-ESO Survey data, there has been no systematic study of the metallicity using spectroscopic analysis (the value of $[\mathrm{Fe} / \mathrm{H}]=-0.11$ dex by Platais et al. 2008 is based on a single star).

In the context of the Gaia-ESO Survey, $\operatorname{Tr} 20$ is the first old OC observed: its proximity (about $3 \mathrm{kpc}$ from the Sun, according to Platais et al. 2008 and Carraro et al. 2010), its mass, its age, and its position (located inside the solar ring in the direction of the Galactic centre) make this cluster very interesting. In fact only very few OCs older than 1 Gyr are known (less than $20 \%$, see e.g., Dias et al. 2002, and web updates), but they are ideal probes for Galactic disc chemical evolution and structure studies (see e.g., Friel 1995; Bragaglia \& Tosi 2006; Magrini et al. 2009; Pancino et al. 2010; Lépine et al. 2011; Yong et al. 2012). Furthermore, few OCs in the inner disc are known. It is fundamental that not only the metallicity and detailed chemistry of $\operatorname{Tr} 20$ are accurately measured, but also its age and distance.

The selection of targets observed by the Gaia-ESO Survey is different for UVES and GIRAFFE instruments: whilst UVES fibres are preferentially allocated to high-probability cluster members, the selection of GIRAFFE targets, mainly based on photometric criteria, is unbiased and high priority is given to all candidate members (see Bragaglia et al., in prep., for more details). One of the goals of the Gaia-ESO Survey is to determine

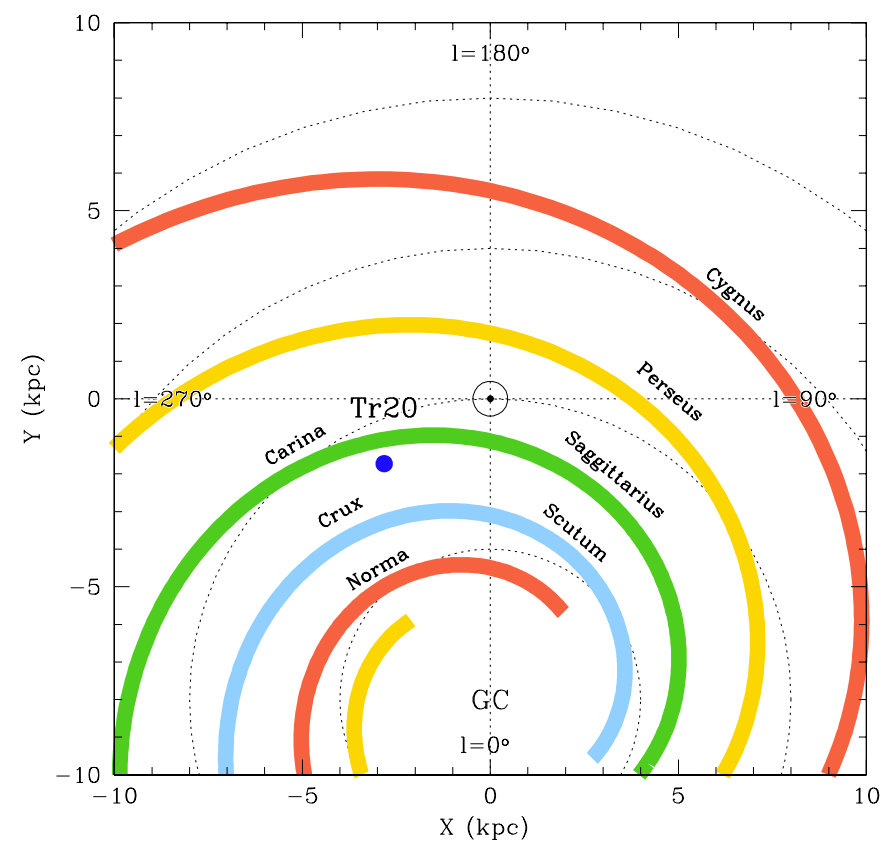

Fig. 1. View of the MW based on the model of Vallee et al. (2005). The position of $\operatorname{Tr} 20$, indicated with a filled blue circle, is based on the results of this analysis. The $\odot$ symbol at the centre of the figure marks the position of the Sun, while the different approximate position of the spiral arms are marked with coloured stripes.

the membership using the radial velocity (RV) and, if possible, exploiting the additional information on the chemical abundances.

The goal of this paper is to determine with better accuracy the cluster parameters from the best-fitting isochrones of the latest evolutionary models, taking into account the effect of differential reddening and the spectroscopic information from the Gaia-ESO Survey for cluster membership and chemical abundance. The GIRAFFE spectra are exploited for determining the RV distribution of the clusters targets, the UVES spectra mainly for the good constraint to the metallicity of the cluster provided by accurate chemical abundances analysis. The large and homogeneous dataset of the survey guarantees a first comprehensive study of several OCs using the same methods. Among intermediate-age and old OCs, Tr 20, NGC 4815 and NGC 6705 (M 11) are also currently being analysed: Magrini et al. (2014) discuss their chemical abundances in general, while NGC 6705 is studied in Cantat-Gaudin et al. (in prep.), and NGC 4815 in Friel et al. (in prep.).

This paper is organised as follows: in the second section we summarise previous studies on Tr 20. In Sect. 3 we briefly describe the photometric and spectroscopic data sets. Sections 4 and 5 describe the analysis of the photometric and spectroscopic data, focusing on the estimate of the differential reddening, the peculiar morphology of the $\mathrm{RC}$, the cluster metallicity and radial velocity distribution. The cluster parameters are described in Sect. 6, while a summary of the whole analysis is presented in Sect. 7.

\section{Trumpler 20: previous studies}

The first study of $\operatorname{Tr} 20$ that defined its evolutionary status using photometry and isochrones was made by McSwain \& Gies (2005, hereafter MS05). Their goal was to determine the fraction of Be stars relative to B stars as a function of cluster age. They used Strömgren photometry and fitted isochrones to identify the 
Table 1. Summary of the parameters estimated for $\operatorname{Tr} 20$ by different authors.

\begin{tabular}{lcccccccc}
\hline \hline Refs. & $\begin{array}{c}\text { Age } \\
(\mathrm{Gyr})\end{array}$ & $\begin{array}{c}{[\mathrm{Fe} / \mathrm{H}]} \\
(\mathrm{dex})\end{array}$ & Method & $\begin{array}{c}(m-M)_{0} \\
(\mathrm{mag})\end{array}$ & $\begin{array}{c}E(B-V) \\
(\mathrm{mag})\end{array}$ & $\begin{array}{c}d_{\odot} \\
(\mathrm{kpc})\end{array}$ & $\begin{array}{c}R_{\mathrm{GC}} \\
(\mathrm{kpc})\end{array}$ & $\begin{array}{c}Z \\
(\mathrm{pc})\end{array}$ \\
\hline MS05 & 0.16 & - & - & 11.92 & 0.26 & 2.42 & 7.05 & 93.82 \\
P08 & 1.30 & -0.11 & $\mathrm{~S}$ & 12.60 & 0.46 & 3.31 & 6.88 & 128.33 \\
S10 & 1.50 & 0.0 & $\mathrm{P}$ & 12.40 & 0.48 & 3.02 & 6.92 & 117.03 \\
C10 & 1.40 & 0.0 & $\mathrm{P}$ & 12.60 & 0.35 & 3.31 & 6.88 & 128.33 \\
\hline
\end{tabular}

B stars in each cluster. For Tr 20 they found only one possible Be star and very few B stars. However, they apparently missed the cluster signatures and fitted a too young isochrone (160 Myr) to the brighter stars, which are more likely field stars.

Platais et al. (2008, hereafter P08) used BVI photometry on a $20^{\prime} \times 20^{\prime}$ field of view, reaching a magnitude of $V=18.5$. The main sequence (MS) of the cluster is clearly visible together with the RC. They estimated an age of 1.3 Gyr using the Padova isochrones (Girardi et al. 2000a) for $Z=0.015$ (the solar composition for this isochrone set is $Z=0.019$ ), a distance modulus of $(m-M)_{0}=12.6 \mathrm{mag}$, and a reddening $E(B-V)=0.46 \mathrm{mag}$. They observed three red giant branch (RGB) and three RC stars with FEROS ( $R=48000)$; five were found to be members based on RV. One of the spectra had a sufficient signal-to-noise ratio $(\mathrm{S} / \mathrm{N})$ to derive stellar parameters and abundances, from which they obtained a metallicity of $[\mathrm{Fe} / \mathrm{H}]=-0.11$.

Seleznev et al. (2010, hereafter S10) derived the cluster parameters and structural parameters using $V$ and $I$ photometry on a field of view of $13.5^{\prime} \times 13.5^{\prime}$, reaching $V \sim 22 \mathrm{mag}$. They estimated an age of $1.5 \mathrm{Gyr}, E(B-V)=0.48 \mathrm{mag}$, and $(m-M)_{0}=12.4 \mathrm{mag}\left(R_{\mathrm{GC}}=7.3 \mathrm{kpc}\right)$ using the Padova isochrones of solar metallicity (Girardi et al. 2000a). They provided a measurment of the radius and centre of the cluster using both their optical photometry and infrared photometry (from the 2MASS catalogue, Skrutskie et al. 2006, for a larger field of view). They derived a radius of $r=5.4^{\prime}$ and centre coordinates $\mathrm{RA}=12: 39: 32$ and $\mathrm{Dec}=-60: 37: 36$.

Carraro et al. (2010, hereafter C10) observed Tr 20 using $U B V I$ filters, deriving the cluster parameters using both twocolour diagrams and CMD. They found an age of $1.4 \mathrm{Gyr}$ from the solar metallicity Padova models (Girardi et al. 2000a). This result is consistent with the one found by P08, but for a different reddening value: the distance modulus $\mathrm{C} 10$ measured is $(m-M)_{0}=12.6 \mathrm{mag}$ and the reddening is $E(B-V)=0.35 \mathrm{mag}$. They explained the visible broadening of the main-sequence turn-off (MSTO or simply TO) with the strong impact from binary systems and the unavoidable contamination by field interlopers. They also discussed the prominent and extended RC of the cluster.

Platais et al. (2012, hereafter P12) estimated the radial velocities of nearly 1000 stars belonging to the upper MS and RC/RGB using the GIRAFFE fibres on the FLAMES instrument at VLT. Neither photometry nor RVs are public. They derived an average $\langle R V\rangle=-40.40 \pm 0.12 \mathrm{~km} \mathrm{~s}^{-1}$ using $68 \mathrm{RC}$ stars. They were able to define 471 cluster members and suggested that about 50 to 100 stars might still be field stars when the statistics on the rotation velocity $v \sin i$ is also taken into account. They also estimated the differential reddening, concluding that it plays a strong role in shaping the cluster CMD morphology. In particular, they ruled out the hypothesis of multiple populations in $\operatorname{Tr} 20$ (which they proposed in earlier works) as an explanation of the broad MSTO.

We summarise the results available in the literature in Table 1, where we list age, metallicity, the method used to derive metallicity (from spectroscopy, S, or photometry, P), the distance modulus and reddening, the distance from the Sun and the Galactic centre $\left(R_{\mathrm{GC}}\right.$ has been computed assuming $R_{\mathrm{GC}, \odot}=$ $8 \mathrm{kpc}$, see Malkin 2013), and the height above the Galactic plane. Regarding the age, a good consensus is obtained within the quoted errors, except for MS05, for the reason explained above. The differences in distance modulus and reddening are related to the age and metallicity adopted and to differences in the photometric data. In the light of the new results obtained within the Gaia-ESO Survey, the metallicity obtained with highresolution spectroscopy is of great importance also to determine the other parameters more accurately and limit the degeneracy between them. We used this information and took into account the differences in photometry and different evolutionary models to derive a reliable estimate of age, distance, and reddening of $\operatorname{Tr} 20$. We also improved the available photometric catalogue, proposing a correction for differential reddening that helps to explain the morphology of the MSTO and better constrain the evolutionary status of the cluster. Moreover, we discuss in detail the peculiar $\mathrm{RC}$, which is too extended with respect to the predictions of standard stellar evolutionary models.

\section{Observational data}

\subsection{Photometry}

We obtained the $B V I$ photometry of P08 for Tr 20 from the CDS ${ }^{1}$ and that of $\mathrm{C} 10$ from WEBDA ${ }^{2}$. By using the CATAPACK ${ }^{3}$ programme, we were able to cross-identify the stars in common between the two catalogues and compare their photometry. The difference between P08 and C10 is on average $-0.052 \pm 0.045$ in $V,+0.057 \pm 0.025$ in $(B-V)$, and $-0.146 \pm 0.032$ in $(V-I)$. Figure 2 shows these offsets and how they scale with magnitude.

Note that our result is different from that in $\mathrm{C} 10$ (their Fig. 3); the $V$ magnitudes and $(B-V)$ colours from the two works roughly agree, but we were unable to reproduce the perfect agreement for $(V-I)$. We have no apparent explanation for this and decided to give preference to the $(B-V)$ colour with respect to the $(V-I)$ one throughout this analysis. Moreover, these differences have an important impact on the derivation of the cluster properties, as discussed in Sect. 6.

\subsection{Spectroscopy}

Many stars of Tr 20 have been observed within the Gaia-ESO Survey, using the GIRAFFE HR15N grating and the UVES $580 \mathrm{~nm}$ setup. The targets were selected to lie on or near the

\footnotetext{
1 The Strasbourg Astronomical Data Center, see http://cds.u-strasbg.fr/

2 The on-line database collecting OC photometry, see webda.physics.muni.cz

3 Made available by Paolo Montegriffo at the INAF Bologna Observatory.
} 

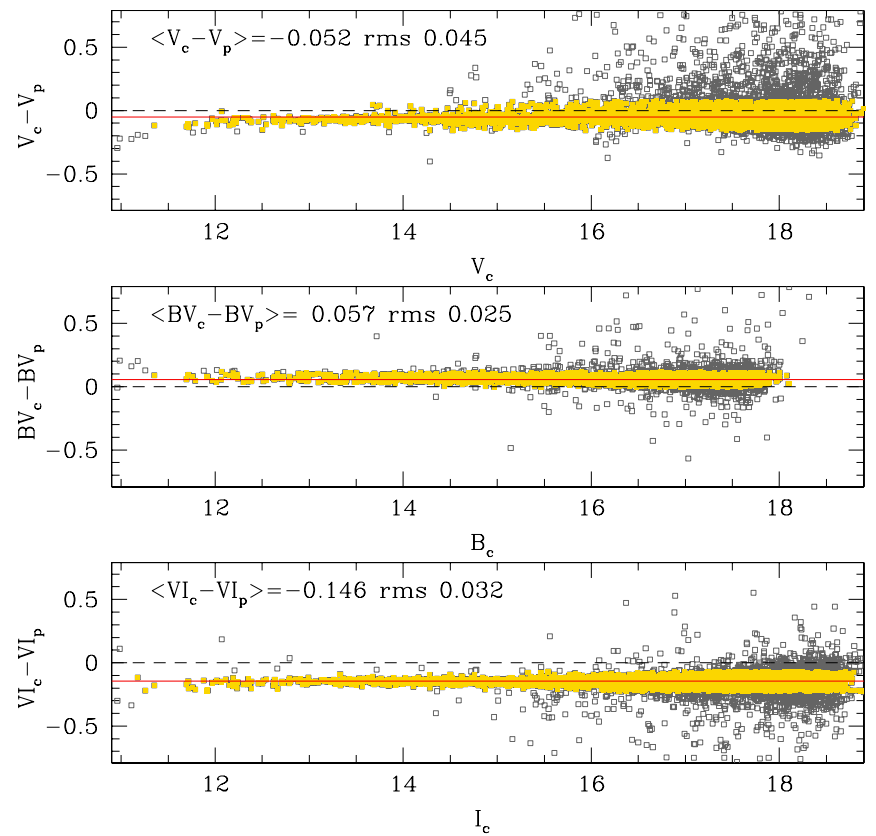

Fig. 2. Systematic differences between the data sets of $\mathrm{P} 08$ and $\mathrm{C} 10$ in $V,(B-V)$, and $(V-I)$. The yellow filled squares were used to compute the average differences.

evolutionary sequences of the CMD. Concerning the cluster MS, stars with colour between the blue envelope of the MS and about 0.3 mag redder were considered potential targets, with a higher priority given to stars closer to the cluster centre. The cluster RC is evident and rich, and all stars in this phase were considered potential targets. Some potential sub giant stars were also taken into account. The GIRAFFE targets were chosen mainly on the MS and on the probable subgiant branch (SGB), the RGB, and the RC, while the UVES targets fall on the RC. In Fig. 3 we show their spatial position and their locus on the CMD. The $\mathrm{S} / \mathrm{N}$ of the spectra depends mainly on the luminosity of the targets, spanning from about 10 for the fainter targets up to 300 for the most luminous. The median $\mathrm{S} / \mathrm{N}$ is about 30 for the GIRAFFE targets, and 60 for those of UVES.

The Gaia-ESO Survey observations of Tr 20 have been obtained in Spring 2012 and 2013. We observed 42 stars on the RC with the UVES fibres, while $527 \mathrm{MS}$ and giant stars were observed with the GIRAFFE fibres. Only 13 UVES targets were fully analysed as part of the first internal data release (GESviDR1Final) of the survey, which encompassed the first six months of observations, while for the other targets a full analysis will be available in the next releases. However, the RV is available for all the Gaia-ESO Survey stars and for the 954 archive spectra taken by Platais and collaborators using the GIRAFFE HR09B grating, as described in P12 and reanalysed inside the Gaia-ESO Survey; this greatly benefits the analysis of the RV distribution described in this paper. We were able to cross-identify 953 of them. In our sample, 40 stars were observed both with UVES and GIRAFFE; 110 stars with the two GIRAFFE setups; 10 with all the setups. More information on the observations is given in Table 2 . In Table 3, only available online, we list the relevant information for all the spectra (obtained with the three different setups HR09B, HR15N, and UVES) of the targets used in this paper. We report the identification number from the $\mathrm{C} 10$ and P08 catalogues, the Gaia-ESO Survey id, RA, and Dec coordinates, the magnitudes $V$ and $B$ from $C 10$ when available, otherwise those from P08, and the RVs.
Table 2. Summary of the GES and public spectroscopic observations for $\operatorname{Tr} 20$.

\begin{tabular}{lccc}
\hline \hline Setup used & $\begin{array}{c}\lambda \lambda \\
(\mathrm{nm})\end{array}$ & $\begin{array}{c}\text { Time exp. } \\
(\mathrm{min})\end{array}$ & \# stars \\
\hline UVES 580 nm & $476.0-684.0$ & $3 \times 50$ & 525 \\
GIRAFFE HR15N & $647.0-679.0$ & $3 \times 50$ & 42 \\
GIRAFFE HR09B & $514.3-535.6$ & $a$ & 954 \\
\hline
\end{tabular}

Notes. ${ }^{(a)}$ ESO public archive data of programme 083.D-0671 (see P12) processed within the GES.

The Gaia-ESO Survey consortium is structured into several working groups, WGs, with specific duties. The data reduction is performed by WG 7 and a comprehensive description of the methods used can be found in Sacco et al. and Gilmore et al. (both in prep.). The RV information, available for all the targets observed with both GIRAFFE and UVES, is determined by WG 8 (see Gilmore et al., in prep.). For the abundance derivation, see Sect. 5; at the moment, only 13 UVES targets are fully analysed and were used here, while those of GIRAFFE will be released in the near future. With this new spectroscopic information it is possible to derive the cluster parameters with unprecedented accuracy.

\section{Photometric analysis}

\subsection{Centre, mass, and radius}

We made a broad photometric selection to remove very obvious field polluters from the sample, keeping the MS, MSTO, and RC stars. We determined the position of the cluster centre by an iterative process using the same method as described in Donati et al. (2012). We computed the barycentre of the positions of the stars, then took the $70 \%$ of stars closest to this position and recomputed their barycentre, and iterated until convergence on a central position. To avoid selecting too many field stars we set magnitude cuts at $V=16,17$, and 18 . This led to similar results because the position we obtain is identical within $0.5^{\prime}$ around the coordinates: $\mathrm{RA}=12^{\mathrm{h}} 39^{\mathrm{m}} 32^{\mathrm{s}} .8$, Dec $=-60^{\circ} 37^{\prime} 37^{\prime \prime} .4$ (or, in Galactic coordinates: $l=301^{\circ} .47, b=2.21$ ).

Tr 20 is densely populated and stands out against the field stars, which enabled us to follow its density profile. C10 indicated that the completeness of their photometry is better than $90 \%$ for magnitudes $V<19$. To be conservative, we only used stars brighter than $V=18$. We performed a least-squares fit of a two-parameter King profile (King 1962),

$f(r)=\rho_{\mathrm{bg}}+\frac{\rho_{0}}{1+\left(r / r_{\mathrm{c}}\right)^{2}}$,

where $\rho_{\mathrm{bg}}$ the background density, $\rho_{0}$ the central density, and $r_{\mathrm{c}}$ the core radius are left as free parameters. The observed profile and the best-fit are shown in Fig. 4. Using a three-parameter King model that also takes into account a tidal radius does not improve the goodness of fit. This means that the tidal radius of $\operatorname{Tr} 20$ is larger than our field of view, and the region where the density profile starts to decrease faster is too far out for our data.

Since the model fitting provides a value for the background stellar density, we were able to remove its contribution to the star counts. The density profile was integrated to obtain the total number of stars contained in the cluster. Assuming a Salpeter mass function and the best-fitting PARSEC isochrone (see Sect. 6), we produced a synthetic population that contains the same number of stars as $\operatorname{Tr} 20$ in the magnitude range 

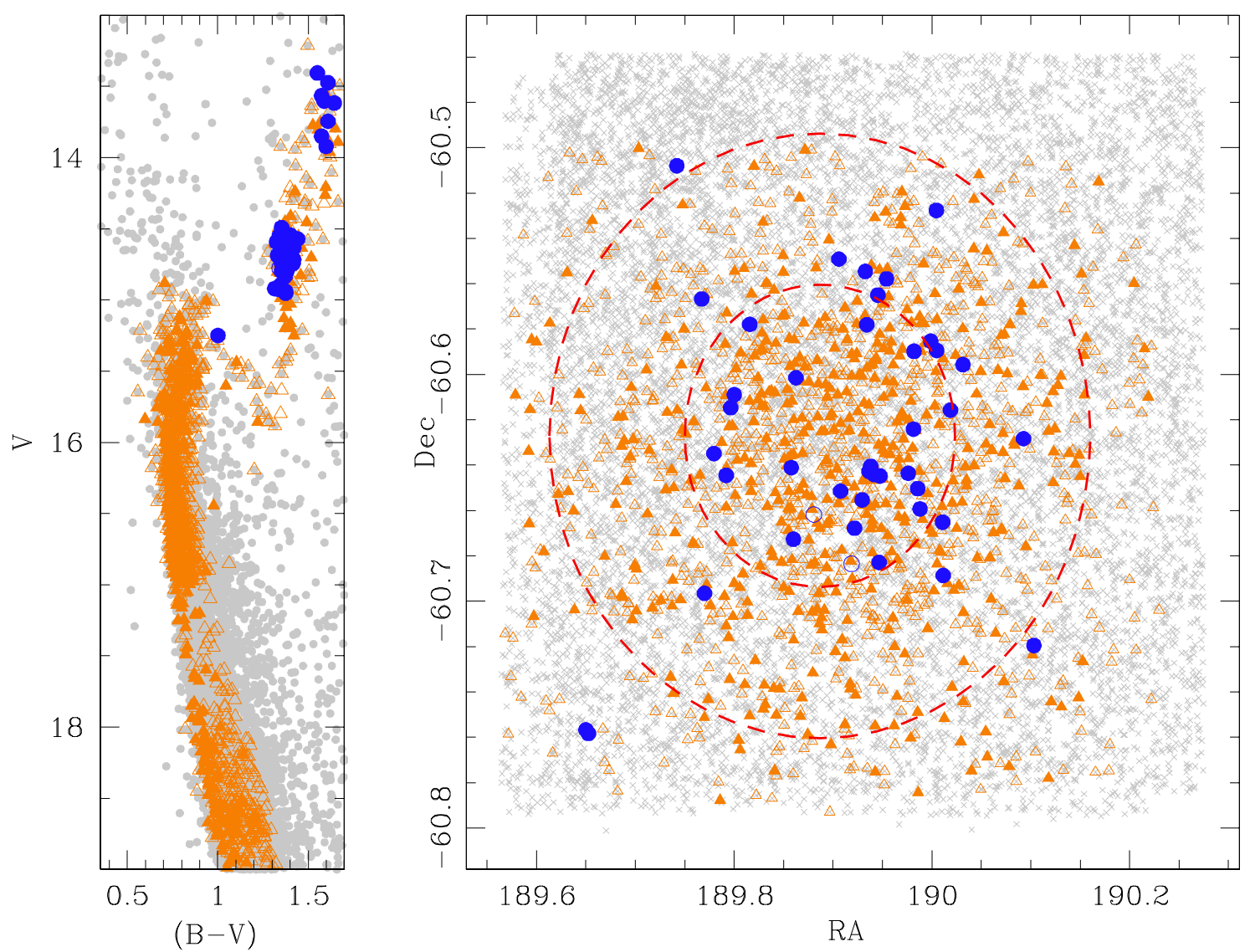

Fig. 3. Left panel: CMD of the GES targets for Tr 20 with a distance from the centre $d<8^{\prime}$. Right panel: spatial distribution of the GES targets; the red dashed circles define the regions for $d<4^{\prime}$ and $d<8^{\prime}$ from the centre. In both panels, the orange triangles are GIRAFFE targets, while the blue filled circles are those of UVES; filled symbols are candidate members for RV (see Sect. 5). The stars in the photometric catalogue are plotted in grey.

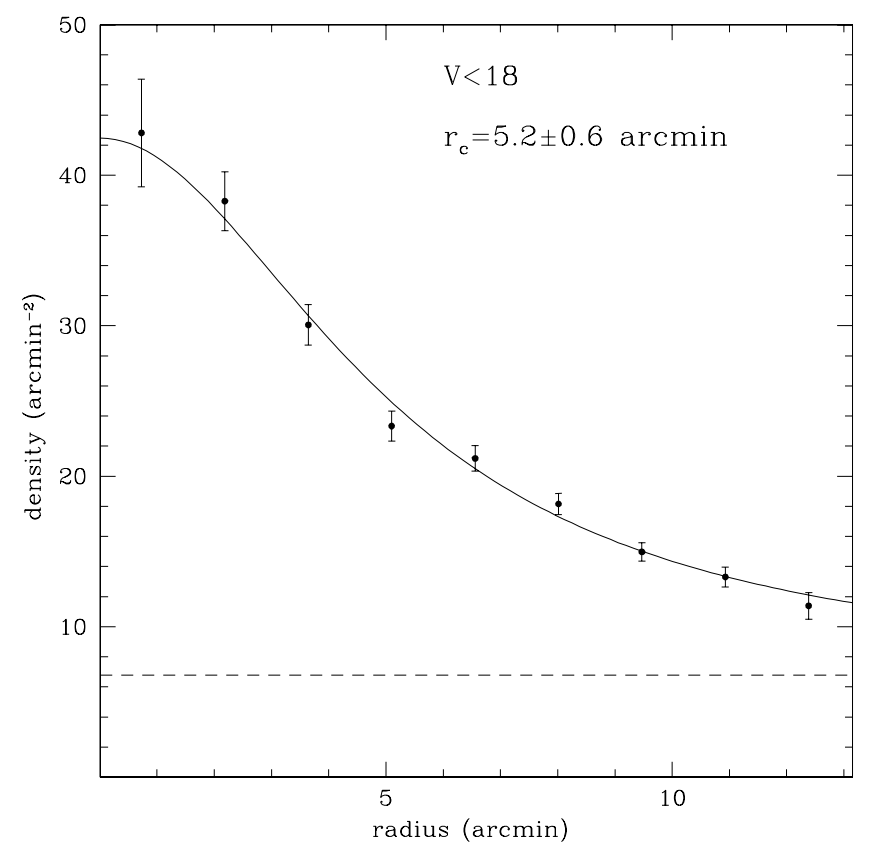

Fig. 4. Density profile of Tr 20 using stars with $V<18$. The error bars are the random errors. The best-fit is a two-parametric King model of core radius $5.2 \pm 0.6$ arcmin.

$V<18$. We added all the masses of stars down to $0.08 M_{\odot}$ and found a total mass of about $6800 M_{\odot}$.
Varying the age of the isochrone within the uncertainties gives an estimate of the error on the total mass. This whole operation was also performed by selecting stars brighter than $V=17$ and $V=16$, which yelded very similar results. Finally, we estimated the total mass of $\operatorname{Tr} 20$ to be $6700 \pm 800 M_{\odot}$. We can draw a more conservative estimate by adding the contribution of the random errors to the star counts. Considering the Poissonian uncertainty, we find masses between the extreme values of 5500 and $8000 M_{\odot}$.

\subsection{Differential reddening}

The position of $\operatorname{Tr} 20$ in the Galactic disc and its high reddening estimates strongly suggest differential reddening (DR, as was discussed, e.g., by P12). The main effect of DR on the CMD appearance is that it broadens the sequences. This is mainly due to the presence of patchy dust structures in the field of view, which cause different extinctions along the line of sight. Photometric errors have a similar effect on the CMD appearance, but the broadened MS of Tr 20 cannot be explained only with errors since they are too small in the two photometric studies considered, as discussed in the original papers.

Other explanations cannot be a priori ruled out. For example, a significant age spread during the star formation process can produce an observed broadening in the TO phase and in later phases, and unresolved binary systems also widen the MS and TO since they have redder colour and brighter luminosity than single objects. A metallicity spread has in principle an effect very 
similar to the DR, even though there is no evidence among the OCs of inhomogeneities in the overall metallicity. Finally, stellar rotation might also affect colour and magnitude; this would make rotating stars seem fainter and redder (see Bastian \& de Mink 2009; Li et al. 2012). Hot early-type stars, such as those near the MSTO, can be fast rotators, as demonstrated by P12 and as seen from the Gaia-ESO Survey GIRAFFE spectra. However, the rotation has a mild effect on the CMD appearance (see P12), confirming the study of Girardi et al. (2011), who excluded rotation as a possible explanation of the extended TOs observed sometimes.

An estimate of the DR for Tr 20 has been performed in P12. They used about 200 slow-rotator stars (located in the upper MS) and evaluated their distance, along the reddening vector direction, from a hand-defined blue envelope of the MS. They smoothed the measurements on a grid using a scale of $1^{\prime}$ and adopting for each bin the median of the nearest few measurements that fell in the same bin of the grid. They demonstrated that the effect of DR on the cluster face is not negligible, but we were unable to use their individual DR values because they are not publicly available at the moment.

We decided to apply a different method of evaluating the DR here. This method is a revision of the one described in Milone et al. (2012) adapted to the case of the OCs, which are less populated and more polluted by field stars than the globular clusters. The main steps of the process are the following:

- we define a fiducial line along the MS to be used as a reference locus for the DR estimate (the choice made for this cluster is described in the following paragraphs);

- we define a region around the fiducial on the MS (we call it MS box for conciseness): all stars falling in this region are used to estimate the DR;

- for each star in the catalogue we pick up its 30 nearest and brightest stars inside the MS box and compute their median distance from the fiducial line in the CMD plane. This distance is used to correct colour and magnitude along the reddening vector direction;

- after the first provisional estimate of the DR is applied star-by-star, we repeat this procedure until convergence is reached. The convergence criterion is a user-defined percentage of stars for which the DR correction estimate is lower than the average rms on these estimates;

- after a final value for the DR is obtained for each star, a binning is performed in the spatial plane. The spatial scale used is compatible with the average distance of the 30 neighbour stars selected and used for the DR estimate. At this point a rejection of outliers is performed: stars for which the DR estimate has an rms higher than average or for which the average distance of the 30 neighbours is larger than average are not taken into account;

- a final and reliable value for the DR is then computed as the average value of the DR corrections associated with the stars falling in the same bin, and the error on this estimate is the associated rms. The DR values obtained in this way are not absolute values, but are relative to the fiducial line.

We used the photometry of $\mathrm{C} 10$ because it reaches fainter magnitudes, so that the MS is well described on a wider magnitude range. We estimated the DR in the $B-V$ colour, since the $\mathrm{C} 10$ and P08 photometric data agree better in this colour than in $V-I$ (see Sect. 3.1). The direction of the reddening vector was derived assuming the standard extinction law $\left(R_{V}=3.1\right)$ described in Dean et al. (1978). The fiducial line was defined using the CMD of the inner part of the cluster (all the stars in

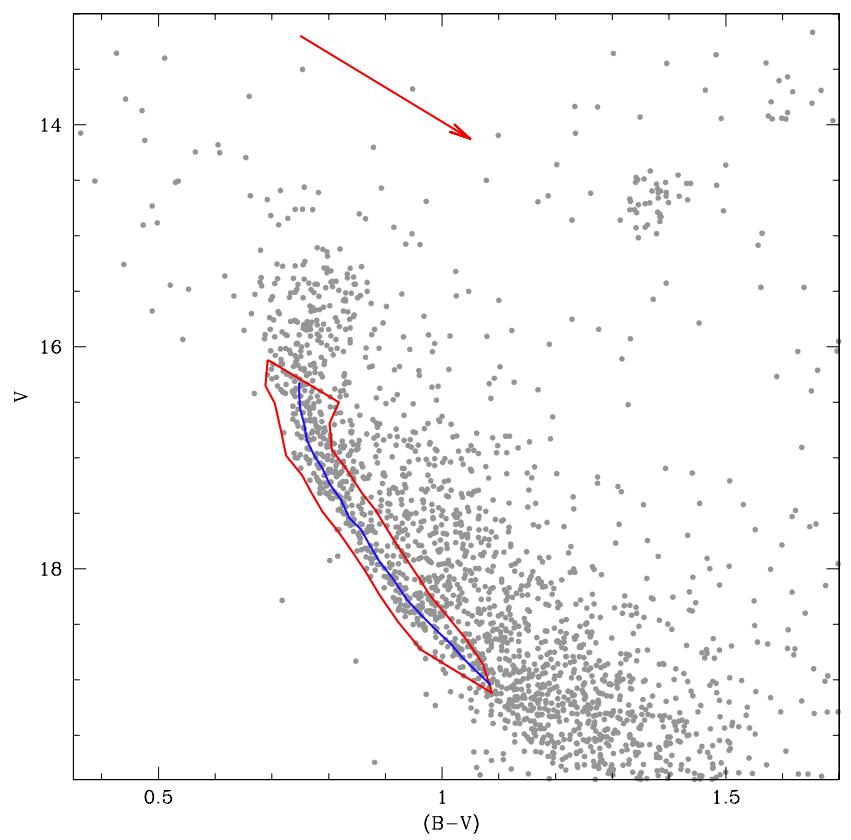

Fig. 5. CMD of $\operatorname{Tr} 20$ inside $4^{\prime}$. The red box and the blue line indicate the MS box and the fiducial line for the DR estimate. The red arrow indicates the reddening vector, both in direction and size.

C10 inside $4^{\prime}$ to clearly identify the cluster signature from the field contamination) and was chosen as the ridge line along the MS. In Fig. 5 the box and the fiducial line used are highlighted. Several attempts were made to avoid fiducial lines that during estimating the DR led to corrections that artificially and noticeably changed the magnitude and colour of the age-sensitive indicators. We aimed to keep the RC, MSTO, and the blue envelope of the MS as close as possible to the observational CMD to limit spurious interpretations of the cluster parameters due to DR corrections. When defining the MS box, we avoided the broadened and curved region of the TO, where the morphology might hamper a correct interpretation, and the fainter part of the MS, where the photometric error is larger.

Taking into account the star counts of the inner and outer parts of the cluster (see Sect. 4.1), we decided to limit the application of correction for DR to stars inside a region of $6^{\prime}$ of radius. For the outer parts the contamination of field stars became significant (the contrast density counts with respect to the field plateau drops below 50\%) and any attempt to estimate the DR was severely affected by field interlopers.

For all the stars inside $6^{\prime}$, a value of DR was computed using the 30 nearest stars falling in the MS box. Then spatial smoothing was applied to obtain a more robust statistic, adopting a binning of $50^{\prime \prime}$ in right ascension and declination.

In Fig. 6 (upper panel) we show the map of the DR obtained in terms of $\Delta E(B-V)$ with respect to the fiducial line. It ranges from about -0.07 to about +0.10 . In particular, a region of low reddening is clearly identifiable. Comparing our results with those presented in Fig. 3 of P12, we obtain qualitatively the same result, with a region of lower DR in the north-western part. We found an excursion in the DR estimates of about 0.15 mag, similar to the $0.1 \mathrm{mag}$ discussed in P12. Our higher value can be explained by the fact that we did not impose a blue envelope, allowing negative correction for DR, while P12 fixed the DR at zero for stars bluer than their reference line. In Fig. 6 (lower panel) we show the corresponding map of the error associated to our estimates. The discrete appearance of these maps is due to 

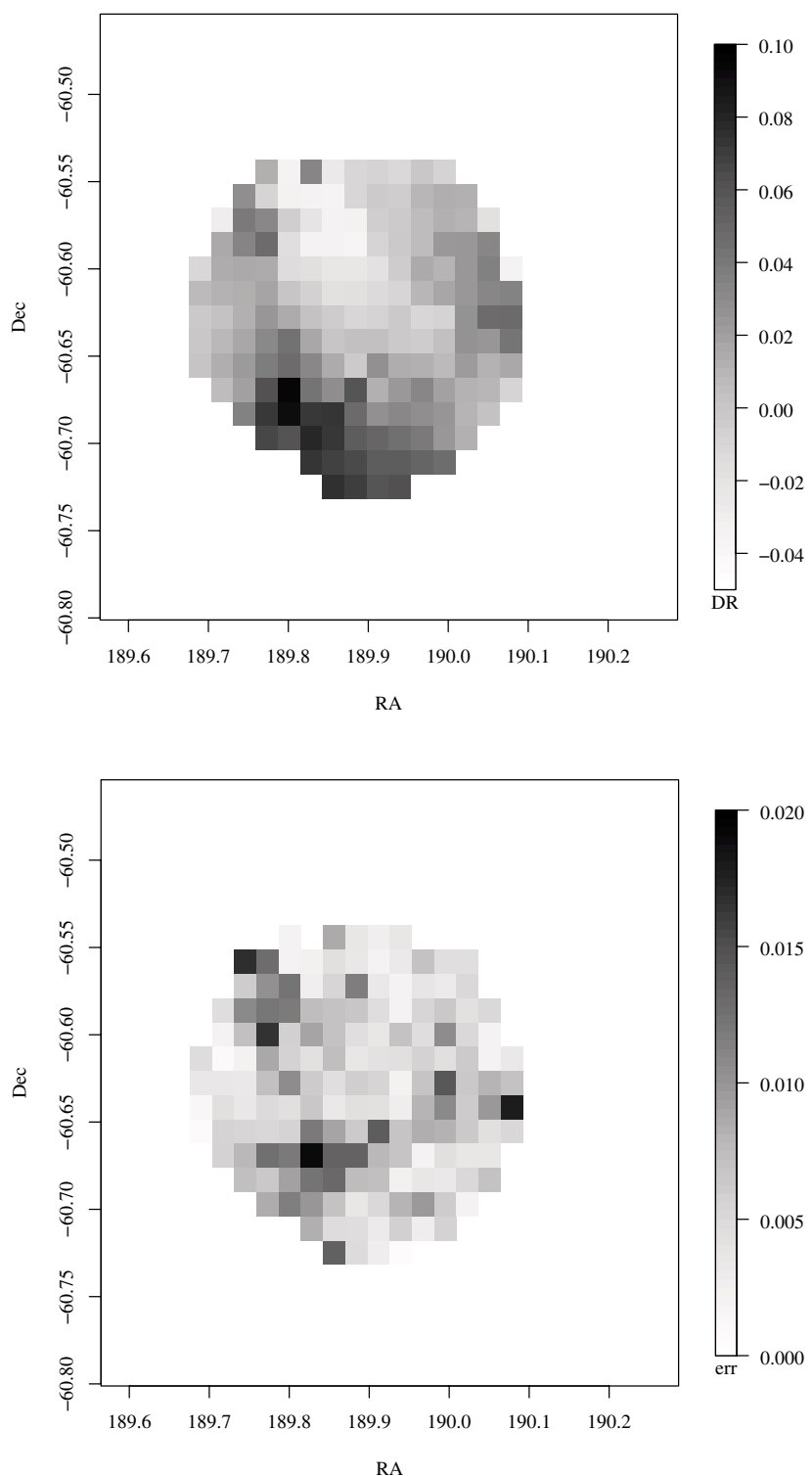

Fig. 6. Upper panel: colour deviations from the reference line due to the effect of DR, mapped on a $50^{\prime \prime} \times 50^{\prime \prime}$ grid for stars inside $6^{\prime}$ from the centre. Lower panel: corresponding error map for the computed colour deviations from the reference line. The grayscale on the right side of each panel indicates the level of each parameter plotted.

two facts: the poorness in sampling a circular area using polygonal bins and the avoidance of interpolation in the corners, where the poor statistics may produce weak estimates. The table with the DR estimates is available through CDS.

The overall effect of the DR on the CMD appearance is shown in Fig. 7. The MS and MSTO regions appear tighter, reducing the broadening of these phases substantially. This improvement is highlighted in black in the figure for the upper MS, but the lower part also benefits from the DR correction. The RC stars are more clumped, highlighting the peculiar morphology of this phase (see Sect. 4.3). Our DR estimate did not change the luminosity level and colour of age-sensitive indicators such as the MSTO, the bright limit of the MS, or the red-hook (RH) phase, limiting artificial estimates of the cluster parameters. The main difference to the method used in P12 is that we used many more stars to estimate the DR, which is therefore supported by a robust statistic. Moreover, we selected stars on the lower MS, avoiding objects at the MSTO. In this part of the CMD other physical

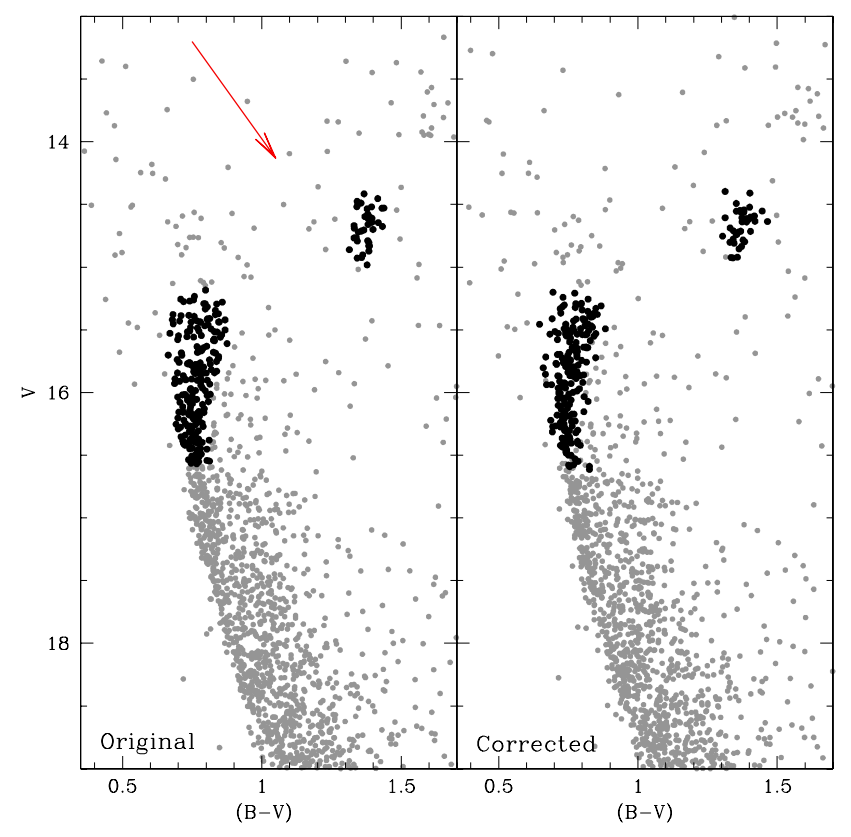

Fig. 7. CMDs for $\operatorname{Tr} 20$ stars (photometry from $\mathrm{C} 10$ ) with distance from the cluster centre $d<4^{\prime}$. Left panel: original photometry. The red arrow shows the direction of the reddening vector. Right panel: photometry corrected for DR. The TO and RC regions are plotted in black to clearly show the effect of the correction for DR.

mechanisms than DR have a more significant impact on the star magnitude and colour (in particular binaries), and, furthermore, the shape of the MS is much more sensitive to the metallicity and age, limiting the accuracy on both the DR estimates and the definition of the fiducial. On the other hand, the P12 method has the advantage of using only cluster members. Even though we have spectra for 1370 stars, we selected only 520 candidate members (see Sect. 5.1) and 100 fall in the MS box. They are still too few for a statistically significant estimate of the DR on the cluster field. However, we can quantify the differences between the two methods for the stars in common. Adopting the same MS box and fiducial, we compared the DR corrections obtained for single stars using our method and that of P12, without applying spatial smoothing. We found that the average difference is -0.004 mag with a dispersion of 0.02 mag. No systematic differences between the two methods were found, but only a low intrinsic dispersion. As final caveat, we stress that photometric errors, undetected binary systems, and residual contamination from the field might affect the DR estimation because they all produce a broadening of the MS. Our results are therefore an upper limit to the DR.

\subsection{Red clump}

$\operatorname{Tr} 20$ has been known to feature an extended $\mathrm{RC}$ at $B-V \simeq 1.45$, spread from $V \cong 14.5$ to $V \cong 15$. The de-reddened photometry of Figs. 7 and 8 shows that this extension is real and is not created by DR, as was found by P12. Furthermore, when DR is taken into account, the double structure of the $\mathrm{RC}$, which has previously been discussed by $\mathrm{C} 10$, becomes more evident (see Fig. 8, upper panel). Two distinct groups of stars are evident, one extended and fainter, centred on about $V \sim 14.8$, the other more luminous, centred on $V \sim 14.5$. We see, however, that the $\mathrm{RC}$ cannot be fitted by a single isochrone (see Sect. 6). Table 4 sums up the spectroscopic properties of the 13 UVES targets that are completely analysed; we list the identification and $B, V$ mag 

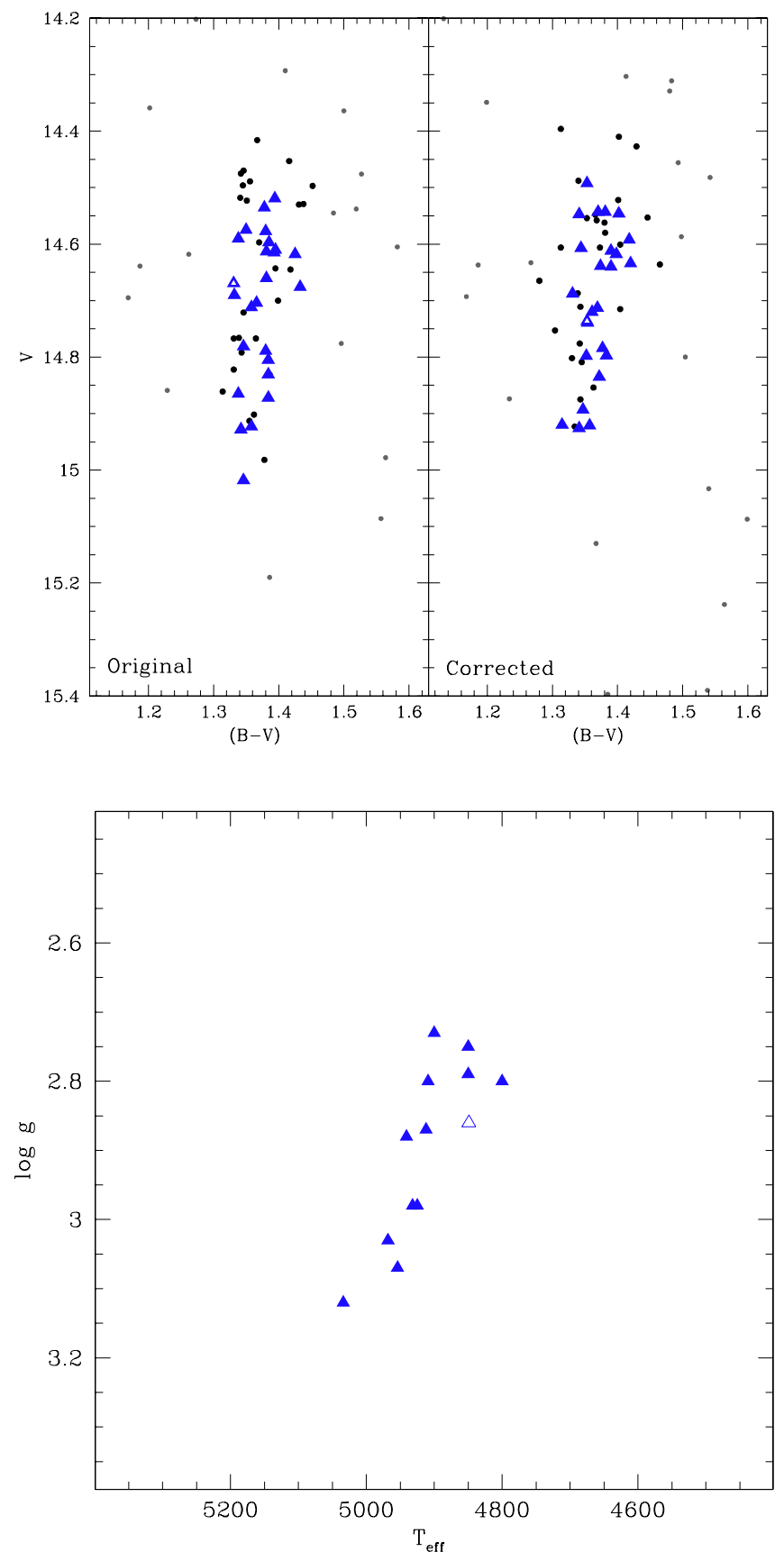

Fig. 8. Upper panel: as in Fig. 7, but for a CMD region centred on the RC position and for stars inside $5^{\prime}$. The blue triangles are the UVES candidate members. The open triangle is the outlier in chemical abundance (see Table 4). Lower panel: the UVES target candidate members in the theoretical $\log g$ vs. $T_{\text {eff }}$ plane (only the 13 stars with complete analysis).

in C10, the Gaia-ESO Survey identifications, coordinates, RVs and the Gaia-ESO Survey atmospheric parameters $T_{\text {eff }}, \log g$, $[\mathrm{Fe} / \mathrm{H}]$, and microturbulent velocity $\xi$ values. The numbers in Table 4 confirm that the observed targets are giant stars, possibly in the RC phase, and are all very good candidate members for RV. Except for one star, which is within $3 \sigma$ from the average metallicity, they also show a remarkable chemical homogeneity, with an average iron abundance $[\mathrm{Fe} / \mathrm{H}]=+0.17$ and a dispersion of only 0.03 dex. In Fig. 8 (lower panel) we show the 13 UVES targets with abundance analysis in the theoretical plane $T_{\text {eff }}, \log g$. They seem to have the same elongated shape as was found in the photometric plane.

Structured RC have been found in other OCs. The works of Mermilliod \& Mayor $(1989,1990)$ and Mermilliod et al. (1997) present about ten intermediate-age MW OCs that show this peculiarity. The first interpretation of these findings, confirmed by the subsequent works of Girardi (1999) and Girardi et al. (2000b), is the possibility that some stars in the RC phase have undergone evolution through helium-core flash, while others have not, because of small differences in the exact core mass. Such differences require a considerable mass spread for clump stars of about $0.2 M_{\odot}$, however, which could in principle result from different mechanisms:

- the natural mass range of core-helium burning stars found in single isochrones, although the current models do not have the level of detail necessary to completely explore this possibility;

- a broad age spread (broader than $100 \mathrm{Myr}$ ), even if never observed in MW OCs;

- star-to-star variations in the mass-loss rates during the RGB phase. Recent asteroseismologic studies on the two OCs NGC 6791 and NGC 6819, the latter of which has an age and metallicity similar to Tr 20 (see Miglio et al. 2012; Corsaro et al. 2012), seem to indicate that no extreme massloss during RGB phases should be expected;

- different stellar rotation history;

- dispersion in the overshooting efficiency in the convective core;

- binarity, if interaction and mass transfer or even mergers are considered.

Nevertheless, the transition between non-degenerate and degenerate He-core ignition would explain the findings in old OCs (age of about 1.4-1.6 Gyr), but not in other OCs, which are too young to be compatible with this evolutionary explanation. $\operatorname{Tr} 20$ is an old cluster (see Sect. 6) and agrees beautifully with this hypothesis. On the other hand, we cannot exclude the shape of the MSTO from this reasoning: even when we remove the effect of DR, a spread is still visible in the CMD that could be due to binary systems or to an age spread (more unlikely); both could give rise to a secondary RC.

Alternatively, Carraro et al. (2011) studied NGC 5822 (0.9 Gyr old) and proposed that part of the stars of its apparently double RC might instead be RGB stars. With the abundance analysis of the UVES targets, the stars in the fainter part of the RC have a $\log g$ too large to be fit by the RC phase of the evolutionary models considered in this work. They might possibly be giant stars in the RGB phase, even if they are too warm for the best-fit model (see Sect. 6, where we derive the cluster parameter with the isochrone fit). In this case, the accurate estimate of lithium abundance might be used to distinguish stars in the RGB phase (lithium should be lower for RC than for RGB stars), but at the moment we do not have this information for our targets.

Understanding the RC of $\operatorname{Tr} 20$ is not easy at all. More investigations are needed to test all the possible hypotheses. About 30 additional giant stars have been observed with UVES, thus it will be possible to obtain a detailed abundance analysis and spectroscopic parameters for a significant fraction of stars that appear in the structured RC of the observational CMDs. Moreover, the Gaia-ESO Survey observations can be used to find binary systems (when combined with archive data), and examine the effect of binaries on the observational CMD quantitatively. 
Table 4. Information for the 13 UVES targets with complete abundance analysis.

\begin{tabular}{|c|c|c|c|c|c|c|c|c|c|c|}
\hline id & GES id & $\begin{array}{c}V \\
(\mathrm{mag})\end{array}$ & $\begin{array}{c}B \\
(\mathrm{mag})\end{array}$ & $\begin{array}{c}\text { RA } \\
(\operatorname{deg})\end{array}$ & $\begin{array}{l}\text { Dec } \\
(\mathrm{deg})\end{array}$ & $\begin{array}{c}\mathrm{RV} \\
\left(\mathrm{km} \mathrm{s}^{-1}\right)\end{array}$ & $\begin{array}{l}T_{\text {eff }} \\
(\mathrm{K})\end{array}$ & $\begin{array}{l}\log g \\
(\operatorname{dex})\end{array}$ & $\begin{array}{c}{[\mathrm{Fe} / \mathrm{H}]} \\
(\mathrm{dex})\end{array}$ & $\begin{array}{c}\xi \\
\left(\mathrm{km} \mathrm{s}^{-1}\right)\end{array}$ \\
\hline 340 & $12391577-6034406$ & 14.67 & 16.00 & 189.8157333 & -60.5779569 & $-40.02 \pm 0.42$ & $4849 \pm 41$ & $2.86 \pm 0.13$ & $0.03 \pm 0.06$ & $1.27 \pm 0.11$ \\
\hline 770 & $12392585-6038279$ & 14.93 & 16.27 & 189.85 & -60.64 & $-42.54 \pm$ & $5034 \pm 106$ & $12 \pm 0.31$ & $0.21 \pm 0.08$ & 0.10 \\
\hline 505 & $12392700-6036053$ & 14.52 & 15.91 & 189.86 & -60.6014733 & $-40.06 \pm 0.42$ & $4800 \pm 77$ & $2.80 \pm 0.24$ & $0.12 \pm 0.07$ & $1.29 \pm 0.10$ \\
\hline 894 & $12393132-6039422$ & 14.77 & 16.11 & 189.8804981 & -60.6617437 & $-35.98 \pm 0.42$ & $4954 \pm 64$ & $3.07 \pm 0.15$ & $0.19 \pm 0.05$ & $1.20 \pm 0.22$ \\
\hline 835 & $12393782-6039051$ & 14.58 & 15.96 & 189.9075746 & -60.6514254 & $-40.53 \pm 0.42$ & $4909 \pm 129$ & $2.80 \pm 0.21$ & $0.21 \pm 0.12$ & $1.30 \pm 0.16$ \\
\hline 346 & $12394419-6034412$ & 14.70 & 16.07 & 189.9341452 & -60.5780773 & $-41.02 \pm 0.42$ & $4941 \pm 90$ & $2.88 \pm 0.23$ & $0.21 \pm 0.07$ & $1.25 \pm 0.06$ \\
\hline 781 & $12394475-6038339$ & 14.61 & 16.01 & 189.9364207 & -60.6427569 & $-39.28 \pm 0.42$ & $4850 \pm 112$ & $2.75 \pm 0.22$ & $0.15 \pm 0.05$ & $1.38 \pm 0.08$ \\
\hline 791 & $12394596-6038389$ & 14.54 & 15.91 & 189.9414399 & -60.6441467 & $-39.69 \pm 0.42$ & $4912 \pm 118$ & $2.87 \pm 0.21$ & $0.20 \pm 0.06$ & $1.27 \pm 0.15$ \\
\hline 287 & $12394690-6033540$ & 14.78 & 16.13 & 189.9453843 & -60.5650194 & $-40.98 \pm 0.42$ & $4968 \pm 77$ & $3.03 \pm 0.10$ & $0.16 \pm 0.11$ & $1.14 \pm 0.14$ \\
\hline 795 & $12394742-6038411$ & 14.71 & 16.07 & 189.9475589 & -60.6447566 & $-39.82 \pm 0.42$ & $4900 \pm 100$ & $2.73 \pm 0.23$ & $0.16 \pm 0.05$ & $1.21 \pm 0.15$ \\
\hline 787 & $12395426-6038369$ & 14.60 & 15.98 & 189.9760346 & -60.6436069 & $-42.45 \pm 0.42$ & $4925 \pm 100$ & $2.98 \pm 0.13$ & $0.17 \pm 0.07$ & $1.36 \pm 0.04$ \\
\hline 399 & $12395975-6035072$ & 14.62 & 16.04 & 189.9988948 & -60.5853363 & $-42.01 \pm 0.42$ & $4850 \pm 87$ & $2.79 \pm 0.19$ & $0.13 \pm 0.07$ & $1.29 \pm 0.11$ \\
\hline 1044 & $12400278-6041192$ & 14.99 & 16.37 & 190.0115263 & -60.6886598 & $-39.04 \pm 0.42$ & $4932 \pm 67$ & $2.98 \pm 0.11$ & $0.16 \pm 0.10$ & $1.37 \pm 0.05$ \\
\hline
\end{tabular}

Finally, we note that double RCs are also evident in clusters of the Magellanic Clouds as shown in Glatt et al. (2008) and Milone et al. (2009) and demonstrated in Girardi et al. (2009). In these high-mass clusters the presence of stars of different age (due to a gap in star formation or a prolonged star formation) looks more probable, similarly to the massive old globular clusters of the MW. However, the transition from non-degenerate and degenerate He ignition is not the universally accepted explanation for them, either. The effect of stellar rotation on the observational CMD (proposed e.g., by Bastian \& de Mink 2009 or Li et al. 2012 and refuted by Girardi et al. 2011) or of binary interactions and merging (e.g. Yang et al. 2011) were considered to reproduce the double RCs.

\section{Spectroscopic analysis}

As we mentioned in Sect. 3.2, the Gaia-ESO Survey data gathering and processing is organised in WGs. In particular, stellar parameters and chemical abundances of F-G-K stars are derived by WG 10 (for a description see Recio-Blanco et al., in prep.) for GIRAFFE, and WG 11 derives them for UVES (Smiljanic et al., in prep.). Earlier-type stars, such as those found near the MSTO in Tr 20, are taken care of separately. Within WG 10 and WG 11 , the analysis is performed by several nodes with different techniques. The common ground for each node is to assume local thermodynamic equilibrium (LTE), using the same model atmospheres (the MARCS models, Gustafsson et al. 2008), the same grid of synthetic spectra (see de Laverny et al. 2012; and Recio-Blanco et al., in prep.), a line list with common atomic parameters (Bergemann et al., in prep.), and a common solar zero point (Grevesse et al. 2007). The results of the nodes are then combined to derive a final set of stellar parameters for each star. With this recommended set of stellar parameters, the nodes recompute the elemental abundances, which are combined to give a set of final values per star. More details can be found in the referenced papers. However, we reiterate that we used only the RVs for the entire spectroscopic sample and the abundances for 13 RC stars observed with UVES.

\subsection{RV distribution}

Using all the RV measurements obtained for the stars observed in $\operatorname{Tr} 20$, it is easy to identify the cluster signature with respect to the field stars. In Fig. 9 we show the RV distribution for the entire Gaia-ESO Survey and archive targets (both UVES and

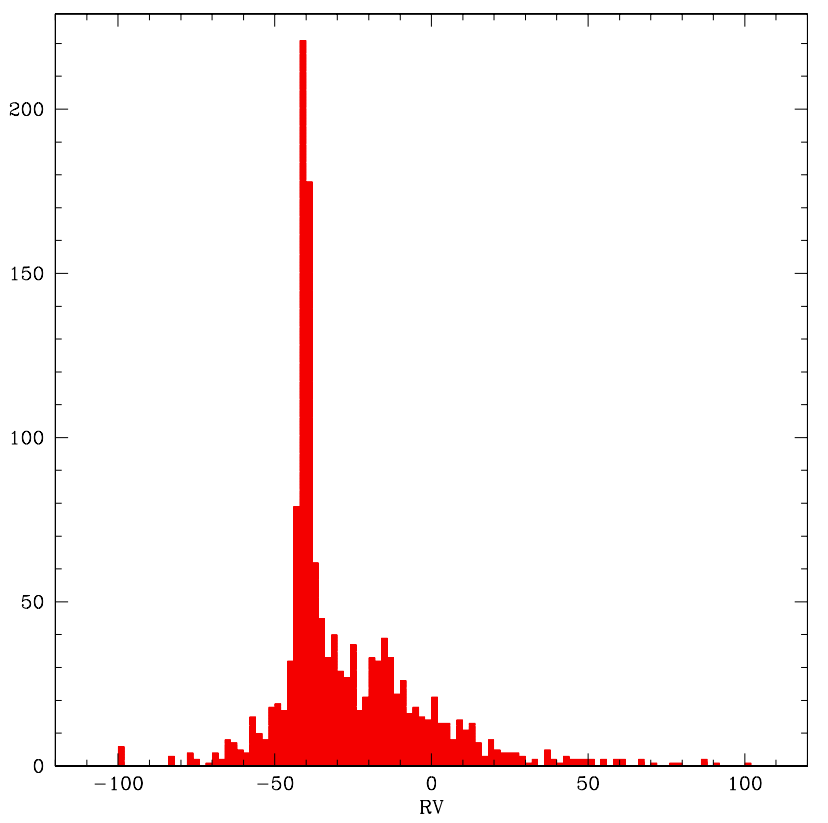

Fig. 9. RV distribution of all the GES targets (1370 stars). The peak of cluster stars is evident at about $-40 \mathrm{~km} \mathrm{~s}^{-1}$.

GIRAFFE). The typical error on the RV for UVES targets is about $0.4 \mathrm{~km} \mathrm{~s}^{-1}$, while it ranges from $0.3 \mathrm{~km} \mathrm{~s}^{-1}$ to several $\mathrm{km} \mathrm{s}^{-1}$ in the worst cases for GIRAFFE targets (see Table 3 ). We used stars in common between the setups to align the GIRAFFE RV estimates to that of UVES, finding that a systematic correction of about $-0.46 \mathrm{~km} \mathrm{~s}^{-1}$ is needed for the HR15N spectra and of $-0.50 \mathrm{~km} \mathrm{~s}^{-1}$ for the HR09B ones. Since we are not interested in the detailed cluster internal dynamics, but only aim to identify candidate member stars, we did not try harder to homogenise the RVs, for instance, by using sky lines to correct for offsets between the zero points of individual spectra. Stars observed with different setups were considered using the following priorities: we used the UVES RV if available, the average RV between GIRAFFE setups when the star was observed with both HR15N and HR09B, and finally the RV derived from only one setup. We estimated the average RV of the sample by selecting stars at different distances from the cluster centre to verify that consistent values were obtained. The inner part of the cluster has of course a higher percentage of cluster members than more distant fields, hence the estimate of the cluster average RV is more 
robust against spurious interlopers. On the other hand, statistics are poorer since the targets are spread up to $12^{\prime}$ from the centre. We decided to choose $8^{\prime}$ as the limiting distance for this analysis.

For each selection made on distances from the centre, outliers were expunged using the following method: the stars whose $\mathrm{RV}$ fell in the smallest RV interval containing 68\% (about the percentage of occurrences inside one standard deviation in a normal distribution) of the RV distribution were retained, then candidates were iteratively selected by using a two-sigma-clipping statistics on the median (five iterations were used). With the last selection convergence was reached and the average and the dispersion were computed. The results of this procedure are shown in Fig. 10 (left panel). We stress here that our aim is to better define the evolutionary sequences on the CMDs by using only the most probable members. A more reliable membership estimation needs a more conservative approach that also considers binaries that might lie outside our selection criteria even if they belong to the cluster.

The values obtained for different selections in distance agree within $0.2 \mathrm{~km} \mathrm{~s}^{-1}$; the estimate obtained in the inner region has a higher dispersion because of the small number of stars. The final values for the average RV and standard deviation used are those obtained for stars inside $8^{\prime}$. The average RV velocity is $\langle\mathrm{RV}\rangle=-40.357 \pm 0.003 \mathrm{~km} \mathrm{~s}^{-1}$ (the rms of the sample used to compute the average RV was $\left.\sim 1.239 \mathrm{~km} \mathrm{~s}^{-1}\right)^{4}$. Considering only the 13 UVES targets with abundance analysis, we obtain an average $\langle\mathrm{RV}\rangle=-40.26 \pm 0.11 \mathrm{~km} \mathrm{~s}^{-1}$, in agreement with the whole sample of stars. For comparison, the average velocity found by P08 is $-40.8 \mathrm{~km} \mathrm{~s}^{-1}$ (based on five stars), while P12 obtained $-40.40 \pm 0.12 \mathrm{~km} \mathrm{~s}^{-1}$ by analysing $68 \mathrm{RC}$ targets. Both agree very well with our values.

Our sample of targets encompasses MS and evolved stars, observed with two instruments of different resolution and spectral coverage. We defined the cluster candidate members using the following simple selection criterion: stars with an RV higher or lower than three times the rms with respect to the cluster average are not considered cluster members. One of the goals of the Gaia-ESO Survey is, in fact, to clean the sequences in the CMDs using membership information and combining GaiaESO Survey and archive spectra. From this we found that $\sim 38 \%$ of the targets (520 stars out of 1370) are good candidate members (see Fig. 10, right panel). According to the Besançon model (see Robin et al. 2003), which was computed for the same coordinates of $\operatorname{Tr} 20$, we estimated that indicatively ${ }^{5}$ about $17 \%$ of the candidate members for RV may still be field stars. As shown in Figs. 3 and 12, almost all the targets located in the SGB region were discarded. The same has been found in P12 (see their Fig. 2), and confirms the expectations from theoretical models. The SGB phase has a very short timescale and is evident in very rich clusters, but poorly populated in smaller clusters. We also found that many stars near the MSTO are not candidate members, as P12 did.

\footnotetext{
4 The quoted dispersion is relative to the sample of stars selected to estimate the systemic velocity of the cluster (411 stars); it is indeed a lower limit of the expected dispersion of the cluster RV because in our analysis we did not consider the effect of binaries, for example.

5 The Besançon model is not appropriate for small scales such as our case, but can still be used to obtain an approximate description of the field contamination.
}
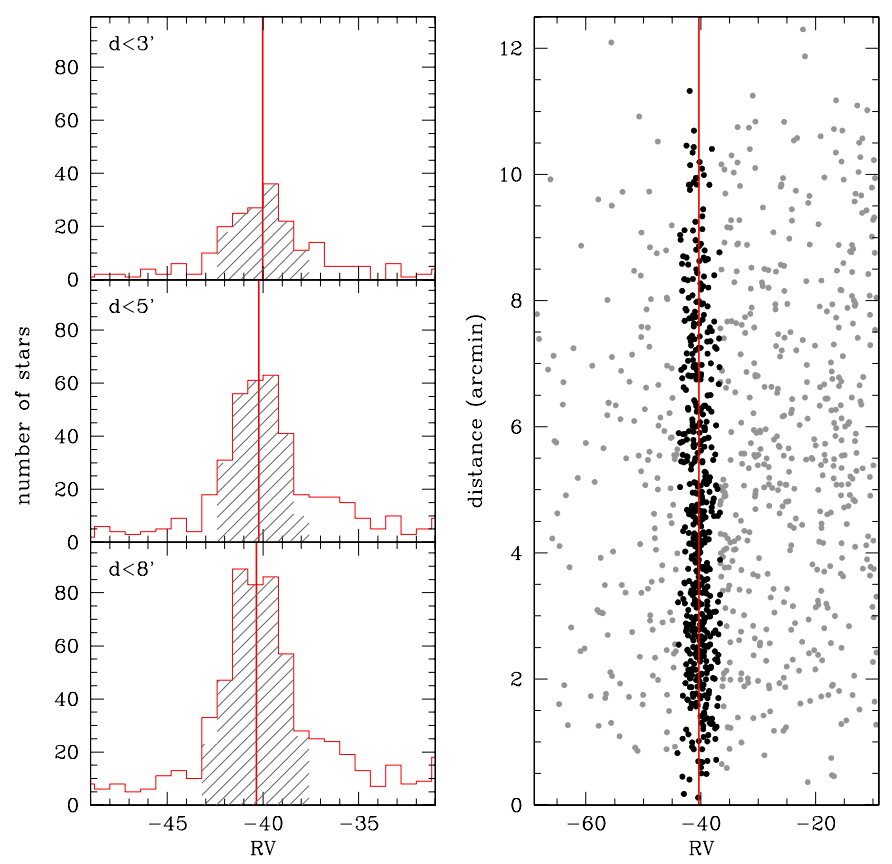

Fig. 10. Left panel: RV distribution of all the GES targets for different distances from the cluster centre. The vertical line is the average RV of stars in the shaded histogram, obtained after an iterative two-sigmaclipping on the median. Right panel: distance from the cluster centre versus RV of the targets. The black dots are the stars selected as candidate members using the average RV (red line) and $\sigma$ obtained from the whole group of targets (bottom histogram, left panel).

\subsection{Cluster metallicity}

In Table 4 we summarise the main results of the abundance analysis for the RC stars (a detailed description is provided in Magrini et al. 2013, where the metallicity and the abundance ratios of four $\alpha$-elements - Mg, $\mathrm{Si}, \mathrm{Ca}$, and $\mathrm{Ti}$ - and of three ironpeak elements - Fe, $\mathrm{Cr}$ and $\mathrm{Ni}$ - are discussed). A metallicity $[\mathrm{Fe} / \mathrm{H}]$ as recommended atmosphere parameter was considered here (see Smiljanic et al., in prep.). In Fig. 11 we show their $[\mathrm{Fe} / \mathrm{H}]$ vs. $T_{\text {eff }}$; there seems to be a mild correlation, even though errors on quantities reduce the statistic significance. This minor effect is irrelevant for the goals of the present work, however, because we used the metallicity information only to confirm the membership and to choose the appropriate isochrones (see next section). All 13 stars are very good candidate members according to their RV (see Sect. 5.1) and have a very low dispersion in metallicity. Only J12391577-6034406 (\#340) shows a slight discrepancy in the iron abundance relative to the other members. The average metallicity is $\langle[\mathrm{Fe} / \mathrm{H}]\rangle=0.17 \mathrm{dex}$ (with a dispersion of $0.03 \mathrm{dex}$ ) without this star, and $\langle[\mathrm{Fe} / \mathrm{H}]\rangle=0.16$ (with a dispersion of 0.05 dex) with it. J12391577-6034406 (\#340) is within $3 \sigma$ from the average. This places $\operatorname{Tr} 20$ in the super solar metallicity regime.

We recall that the solar abundances we adopted are those of Grevesse et al. (2007), that is the iron abundance of the Sun is 7.45. Since this is the same value as adopted by P08, our metallicity is significantly higher than their estimate of -0.11 dex, which was based on only one RGB star. Unfortunately, this star is not in common with the Gaia-ESO Survey UVES targets, which were all chosen to lie on the RC; this prevents a direct comparison and investigation of systematics between the different analyses. On the other hand, the star analysed by P08 is located in the upper part of the RGB, where 


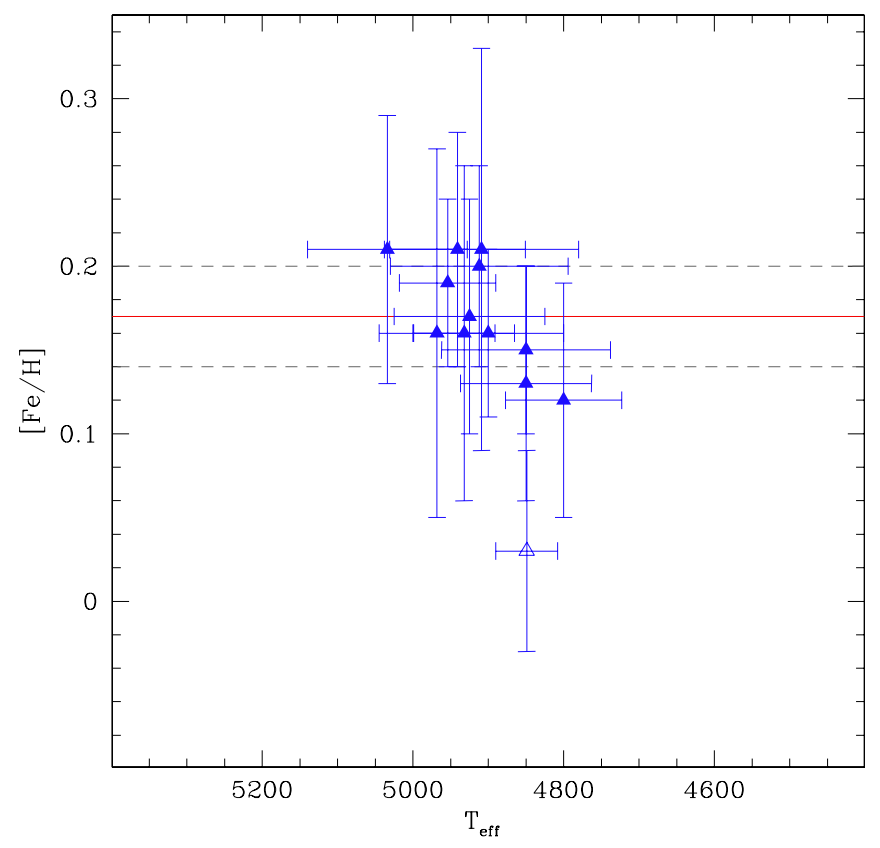

Fig. 11. UVES targets in the $T_{\mathrm{eff}},[\mathrm{Fe} / \mathrm{H}]$ plane. The red line is the average metallicity; the dashed lines define the confidence interval.

1D atmospheric models have more difficulties in reproducing real stars. In the past it has already been found that for low gravity and temperature the abundance analysis leads to lower iron abundances than for RC stars (see, e.g., Friel et al. 2003; Carretta et al. 2005, for a few examples).

\section{Cluster parameters}

By means of the considerable improvements obtained i) in the photometry with the DR estimation; ii) in the metallicity measurement with the high-resolution spectroscopy; and iii) in the membership with RV determinations, we derived the age, distance, and average reddening of $\operatorname{Tr} 20$ by using the classical approach with isochrone fitting. We adopted three different sets of isochrones to have a less model-dependent solution for the cluster parameters: the PARSEC (Bressan et al. 2012), the BASTI (Pietrinferni et al. 2004), and the Victoria-Regina (VandenBerg et al. 2006) isochrones.

The best-fitting isochrone was chosen by eye examination as that which can describe the main age-sensitive evolutionary phases at the same time: the luminosity and colour of the MSTO, $\mathrm{RH}$, and $\mathrm{RC}$ when possible. We used the metallicity resulting from the spectroscopic estimate, that is $[\mathrm{Fe} / \mathrm{H}]=+0.17$, and we converted it to $Z$ taking into account the different solar abundances of the three sets of isochrones. The errors on the estimated parameters are mainly due to the uncertainties in the definition of the age indicators. In particular, the RC has a very peculiar morphology (see Sect. 4.3), which drives the main uncertainty on the age. The photometric error does not have a significant impact on the error budget, apart from systematic ones that can stem from the photometric data reduction and calibration. For instance, the differences found in magnitude and colour between P08 and C10 have an impact on the determination of distance modulus and reddening that will be discussed. For homogeneity we derive the cluster parameters using the catalogue from $\mathrm{C} 10$ corrected for DR in the $B-V$ colour and discuss the effect of the offset between the photometric data. In Fig. 12 we show the results of the best fits, summarised in Table 5 .
For the PARSEC set (see Fig. 12, left panel), for which $\mathrm{Z}_{\odot}=$ 0.015 , we adopted $Z=0.022$. We found that the best age estimate is $1.66 \pm 0.2 \mathrm{Gyr}$, with a reddening $E(B-V)=0.32 \pm 0.02$ mag, and a true distance modulus $(m-M)_{0}=12.64 \pm 0.1 \mathrm{mag}$. The luminosity and colour of the RC are well reproduced, as are those of the RH and upper MS. As has often been found, the lower part of the MS, for $V>18$, is slightly redder in the model than in the observations.

For the BASTI isochrones (see Fig. 12, middle panel) there is a coarse grid in chemical composition, hence we chose two different metallicities, $Z=0.019$ (i.e. solar) and $Z=0.03$, which bracket our spectroscopic metallicity. The best-fit of RH and $\mathrm{RC}$ is obtained for an age of $1.35 \pm 0.2$ Gyr. The more metal-rich solution reproduces the RGB and RC phases slightly better, hence the adopted parameters are from this isochrone: $E(B-V)=0.31 \pm 0.02$ and $(m-M)_{0}=12.72 \pm 0.1$.

The Victoria-Regina isochrones do not include the evolved phases after the RGB for the age of $\operatorname{Tr} 20$. Therefore we chose the best-fitting solution as the one that best matches the MS and MSTO morphologies (see Fig. 12, right panel). Moreover, it is only possible to use a coarse grid in terms of metallicity, hence we tried two different metallicity values that bracket our estimate: $[\mathrm{Fe} / \mathrm{H}]=0.13$ and $[\mathrm{Fe} / \mathrm{H}]=0.22$. They both fairly well reproduce the MS ridge-line for an age of $1.46 \pm 0.2 \mathrm{Gyr}$ and describe the bending at the MSTO and the slope of the lower MS very well, although we prefer that of the lower metallicity because its RGB is closer to the observations, while the latter has a redder RGB phase. The average reddening and distance modulus for this isochrone are $E(B-V)=0.35 \pm 0.02 \mathrm{mag}$, and $(m-M)_{0}=12.70 \pm 0.1 \mathrm{mag}$. With respect to other sets we found that this one reproduces the MS better, though the colour of the RGB is redder than observed. Furthermore, the age is more loosely constrained, since we lack the RC phase.

We repeated this analysis with the Dartmouth isochrones (Dotter et al. 2008) and reached the same conclusions, but for brevity we decided not to detail the analyses as for the other evolutionary tracks. In summary, we found a nice agreement in average reddening and distance modulus for all the model sets. They are similar to what was found by $\mathrm{C} 10$. We set the age in the range from 1.35 Gyr to $1.66 \mathrm{Gyr}$.

Using instead the P08 data, we expect to find some differences in distance modulus and reddening, because the age is mainly constrained by the magnitude difference between the TO and RC luminosities. We found that $E(B-V)$ is about $0.05 \mathrm{mag}$ lower and the distance modulus consequently 0.16 mag higher, which translates into a larger heliocentric distance (by about $0.3 \mathrm{kpc}$ ) and a greater height above the MW disc (about $10 \mathrm{pc}$ ). The differences with the P08 estimates arise because they used stellar models with subsolar metallicity (see Table 1); in particular, this explains their higher reddening.

The results obtained with the three sets are given in Table 5, where we indicate age, distance modulus, reddening, distance from the Sun and the Galactic centre (the $R_{\mathrm{GC}, \odot}$ adopted is $8 \mathrm{kpc}$, see Malkin 2013), distance from the Galactic plane, and mass of the stars at the MSTO. In parenthesis we quote the systematic errors as an additional uncertainty due to the zero points between the photometric catalogues of $\mathrm{C} 10$ and P08.

With the 13 UVES targets it is possible to evaluate the agreement between the models and the data in the theoretical plane $T_{\text {eff }}, \log g$ for the first time for this cluster. In Fig. 13 we show the best-fitting isochrone for the PARSEC set (continuous line): the RC phase of the model fits the upper clump of stars quite well, while the lower elongated group cannot be fitted by any age at this metallicity. In the same figure we show for 
Table 5. Results, errors, and estimated systematic uncertainties using different evolutionary models with $[\mathrm{Fe} / \mathrm{H}] \simeq+0.17$.

\begin{tabular}{lccccccc}
\hline \hline Model & $\begin{array}{c}\text { Age } \\
(\mathrm{Gyr})\end{array}$ & $\begin{array}{c}(m-M)_{0} \\
(\mathrm{mag})\end{array}$ & $\begin{array}{c}E(B-V) \\
(\mathrm{mag})\end{array}$ & $\begin{array}{c}d_{\odot} \\
(\mathrm{kpc})\end{array}$ & $\begin{array}{c}R_{\mathrm{GC}} \\
(\mathrm{kpc})\end{array}$ & $\begin{array}{c}z \\
(\mathrm{pc})\end{array}$ & $\begin{array}{c}M_{\mathrm{TO}} \\
\left(M_{\odot}\right)\end{array}$ \\
\hline PARSEC & $1.66 \pm 0.2$ & $12.64 \pm 0.1(0.2)$ & $0.32 \pm 0.02(0.05)$ & $3.37(0.3)$ & $6.87(0.02)$ & $130.07(10)$ & 1.8 \\
BASTI & $1.35 \pm 0.2$ & $12.72 \pm 0.1(0.2)$ & $0.31 \pm 0.02(0.05)$ & $3.50(0.3)$ & $6.86(0.02)$ & $134.95(10)$ & 1.9 \\
VICTORIA & $1.46 \pm 0.2$ & $12.70 \pm 0.1(0.2)$ & $0.35 \pm 0.02(0.05)$ & $3.47(0.3)$ & $6.86(0.02)$ & $133.71(10)$ & 1.9 \\
\hline
\end{tabular}

PARSEC

BASTI

VICTORIA-REGINA

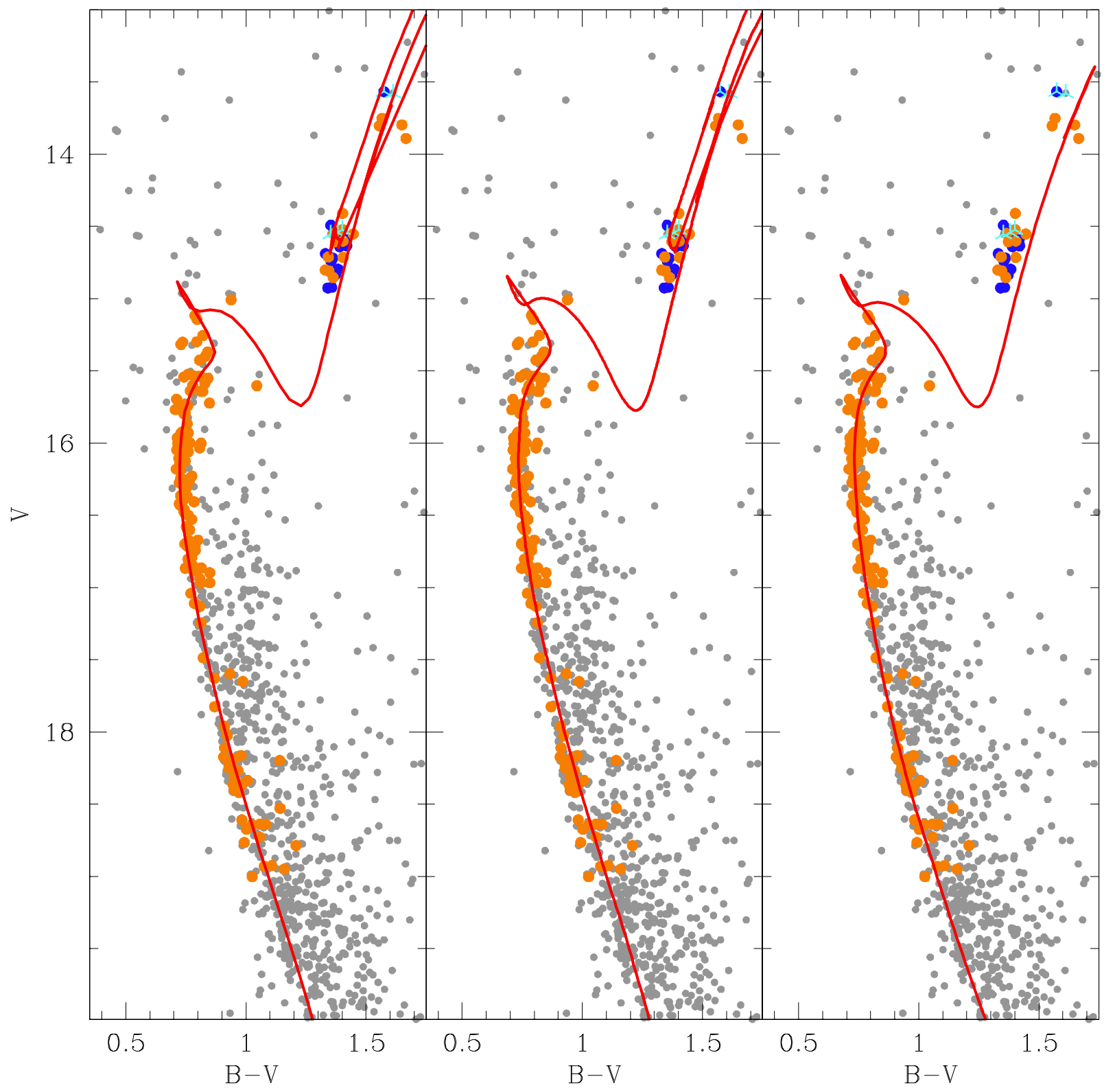

Fig. 12. CMD obtained for stars inside $3^{\prime}$ using the photometry from $\mathrm{C} 10$ corrected for DR, and the best isochrone fit for different evolutionary models (PARSEC - left; BASTI - middle; Victoria-Regina - right). GES target non-members have been disregarded, while members are highlighted with orange (GIRAFFE) and blue (UVES) points. Cyan points are P08 members. See Table 5 for the adopted parameters for the isochrone fitting.

comparison a younger and an older age isochrone (dashed and dot-dashed lines, respectively), for which the clump phase is located at lower $\log g$ with respect to the data, and never reaches $\log g>3$. These stars might be RGB stars (even if still too warm for the best-fitting model) instead of RC stars because they appear in the observational CMD. We discuss the peculiar structure of the RC of Tr 20 in detail in Sect. 4.3.

\subsection{Fitting $B-V$ and $V-I$}

Multi-band photometry can be used to estimate the expected cluster metallicity; in principle, the correct metallicity is the one that produces a good fit with the same isochrone in two different colours with the same parameters (e.g. Tosi et al. 2007). It is very interesting to compare the metallicity obtained from photometry 


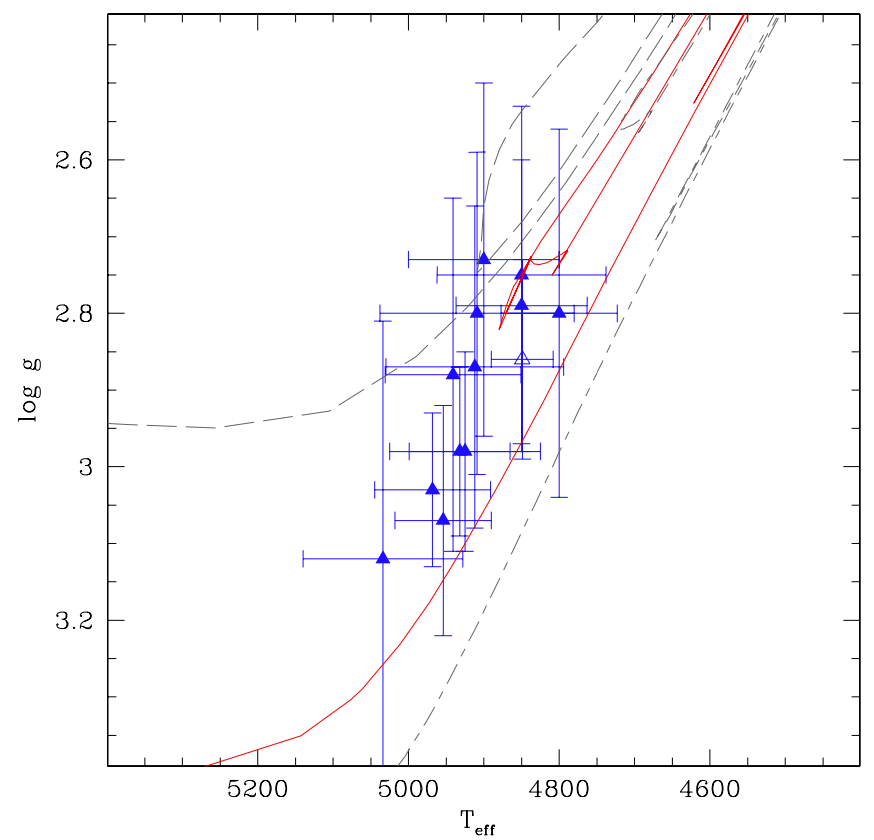

Fig. 13. Blue triangles are UVES targets. The red line is the PARSEC isochrone for the age 1.66 Gyr. For younger ages (dashed line, age of $0.6 \mathrm{Gyr}$ ) or older ones (dot-dashed line, age of $2.5 \mathrm{Gyr}$ ) the RC phase moves toward lower $\log g$ values with respect to the data.

with the one estimated from spectroscopy. Unfortunately, by applying the standard extinction law to convert $E(B-V)$ to $E(V-I)$ $(E(V-I)=1.25 \times E(B-V))$, we obtain a poor fit in $V-I$ for the spectroscopic metallicity, after the cluster parameters are fixed in $B-V$. In Fig. 14 we show the "best-fitting" isochrone in the $V, V-I$ plane (C10 photometry) after applying the standard extinction law. The same inconsistencies hold, but in the opposite way, for the P08 photometry. We tried to find the photometric metallicity that allows a match in both colours. For C10 we derived with a very high metallicity, $Z=0.05$ or $[\mathrm{Fe} / \mathrm{H}] \sim 0.5$, which seems implausible even for a cluster in the inner disc such as $\operatorname{Tr} 20$. For the photometry of P08 a match was obtained with subsolar metallicity, $Z=0.01$ or $[\mathrm{Fe} / \mathrm{H}] \sim-0.18$ dex. Both metallicities are in contrast with the accurate spectroscopic value discussed in this paper.

On the other hand, since $\operatorname{Tr} 20$ is located in the disc between spiral arms, it might be that the standard extinction law is no longer a good approximation. Using a different relation, such as $E(V-I)=1.62 \times E(B-V)$ (see Cardelli, Clayton, \& Mathis $1989)$, the agreement in the case of C10 photometry worsens, while for P08 we obtain a good match for the PARSEC and BASTI isochrones, but not for the Victoria isochrones.

This failure in simultaneously fitting the CMDs in two colours has been found in other cases (see e.g., Ahumada et al. 2013), but no definitive conclusion has been reached. Moreover, in our case we cannot firmly explain the poor match of stellar models in the $B-V$ and $V-I$. One answer can be the already known problem of the photometric transformations from the theoretical to the observational plane. The three models use different transformations, which adds a source of uncertainties to these comparisons. We cannot exclude problems related to the calibration of the photometric data, however, because the catalogues show systematic differences (Fig. 2). Lacking better choices, we decided to constrain our analysis by only using the $B-V$ photometry (for which $\mathrm{C} 10$ and P08 agree better) and the metallicity from the Gaia-ESO Survey.

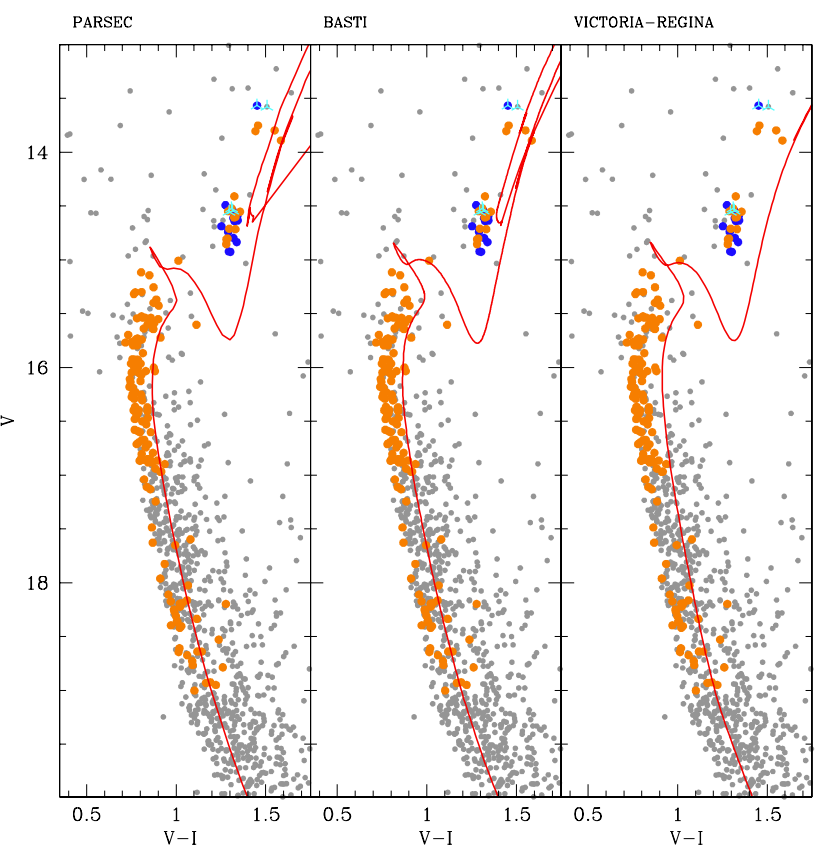

Fig. 14. Same as in Fig. 12, but in the $V$ and $V-I$ CMD, after the standard extinction law has been applied for the isochrones.

\section{Summary and conclusions}

We used available photometry from the literature and spectroscopic Gaia-ESO Survey data to make a comprehensive study of $\operatorname{Tr} 20$ as a pilot analysis for all the old OCs in the GaiaESO Survey. We derived the cluster structural parameters and estimated the effect of DR for the inner part of the cluster. We found that extinction can vary significantly across the field of the cluster, with a range of more than $0.1 \mathrm{mag}$ in $E(B-V)$. The accurate abundance analysis of the Gaia-ESO Survey highresolution spectra of 13 stars in the $\mathrm{RC}$ phase gives an average cluster metallicity of $[\mathrm{Fe} / \mathrm{H}]=+0.17$. From the RV distribution of 1370 stars we estimated the average radial velocity of the cluster, which is $\langle R V\rangle=-40.36 \mathrm{~km} \mathrm{~s}^{-1}$, and defined the candidate member stars on the basis of their RV. With this information we were able to partially clean the catalogue of obvious nonmember stars.

With this information and using the $\mathrm{C} 10$ photometry we estimated the age, distance, and reddening of the cluster by means of the classical isochrone-fitting approach. Using different models (PARSEC, BASTI, and Victoria-Regina), we found a cluster age in the range of $1.35-1.66 \mathrm{Gyr}$, an average reddening $E(B-V)$ in the range $0.31-0.35 \mathrm{mag}$, and a distance modulus $(m-M)_{0}$ in the range 12.64-12.72 mag. Had we used the P08 photometry, these values would be $0.05 \mathrm{mag}$ lower in $E(B-V)$ and $0.16 \mathrm{mag}$ higher in $(m-M)_{0}$. This demonstrates the influence of using different photometric data sets, even when they are apparently of good quality. Only homogeneity can provide the best (internally consistent) parameters and avoid systematics. We cannot fit both $B-V$ and $V-I$ with the same model (with any photometric set) for metallicities that reasonably agree with the spectroscopic one, a problem possibly related to the photometric transformations adopted for the theoretical isochrones or to systematic errors in the photometry of the two data sets.

Our parameters agree reasonably well with most literature values, but were derived through a more robust method. We have discussed the problems of the RC of Tr 20, which cannot be fitted by a single isochrone, but have found no firm conclusion. More 
solid results will be obtained with the next data release, where all the Gaia-ESO Survey spectra of this cluster (especially the highresolution UVES spectra) will be analysed producing a better measurement of the chemical abundances and metallicity and a deeper insight into the properties of the stars that appear to be in the RC phase. This feature is interesting and deserves more investigation.

Acknowledgements. This research has made use of the WEBDA database, originally developed by J.-C. Mermilliod, now operated at the Department of Theoretical Physics and Astrophysics of the Masaryk University, and of BaSTI web tools. Extensive use has been made of NASA's Astrophysics Data System Bibliographic Services, and of the SIMBAD database and VizieR catalogue access tool, CDS, Strasbourg, France. The results presented here benefitted from discussions in three Gaia-ESO workshops supported by the European Science Foundation through the Gaia Research for European Astronomy Training Research Network Program (Science meetings 3855, 4127 and 4415). We acknowledge the support from INAF and Ministero dell'Istruzione, dell'Università e della Ricerca (MIUR) in the form of the grant "Premiale VLT 2012". This work was partially supported by the Gaia Research for European Astronomy Training (GREAT- ITN) Marie Curie network, funded through the European Union Seventh Framework Programme [FP7/2007-2013] under grant agreement no. 264895. D.G. gratefully acknowledges support from the Chilean BASAL Centro de Excelencia en Astrofisica y Tecnologias Afines (CATA) grant PFB-06/2007. I.S.R. gratefully acknowledges the support provided by the Gemini-CONICYT project 32110029 . This work was partly supported by the European Union FP7 programme through ERC grant number 320360 and by the Leverhulme Trust through grant RPG-2012-541. We thank Paolo Montegriffo (INAF, Osservatorio Astronomico di Bologna, Italy) for his software CataPack.

\section{References}

Ahumada, A. V., Cignoni, M., Bragaglia, A., et al. 2013, MNRAS, 430, 221 Bastian, N., \& de Mink, S. E. 2009, MNRAS, 398, L11

Bragaglia, A., \& Tosi, M. 2006, AJ, 131, 1544

Bressan, A., Marigo, P., Girardi, L., et al. 2012, MNRAS, 427, 127

Cardelli J. A., Clayton G. C., \& Mathis J. S. 1989, ApJ, 345, 245

Carraro, G., Costa, E., \& Ahumada, J. A. 2010, AJ, 140, 954

Carraro, G., Anthony-Twarog, B. J., Costa, E., Jones, B. J., \& Twarog, B. A. 2011, AJ, 142, 127

Carretta, E., Bragaglia, A., Gratton, R. G., \& Tosi, M. 2005, A\&A, 441, 131 Corsaro, E., Stello, D., Huber, D., et al. 2012, ApJ, 757, 190

de Laverny, P., Recio-Blanco, A., Worley, C. C., \& Plez, B. 2012, A\&A, 544, A126

Dean, J. F., Warren, P. R., \& Cousins, A. W. J. 1978, MNRAS, 183, 569
Dias, W. S., Alessi, B. S., Moitinho, A., \& Lépine, J. R. D. 2002, A\&A, 389, 871

Donati, P., Bragaglia, A., Cignoni, M., Cocozza, G., \& Tosi, M. 2012, MNRAS, 424, 1132

Dotter, A., Chaboyer, B., Jevremović, D., et al. 2008, ApJS, 178, 89

Friel, E. D. 1995, ARA\&A, 33, 381

Friel, E. D., Jacobson, H. R., Barrett, E., et al. 2003, AJ, 126, 2372

Gilmore, G., Randich, S., Asplund, M., et al. 2012, The Messenger, 147, 25

Girardi, L. 1999, MNRAS, 308, 818

Girardi, L., Bressan, A., Bertelli, G., \& Chiosi, C. 2000a, A\&AS, 141, 371

Girardi, L., Mermilliod, J.-C., \& Carraro, G. 2000b, A\&A, 354, 892

Girardi, L., Rubele, S., \& Kerber, L. 2009, MNRAS, 394, L74

Girardi, L., Eggenberger, P., \& Miglio, A. 2011, MNRAS, 412, L103

Glatt, K., Grebel, E. K., Sabbi, E., et al. 2008, AJ, 136, 1703

Grevesse, N., Asplund, M., \& Sauval, A. J. 2007, Space Sci. Rev., 130, 105

Gustafsson, B., Edvardsson, B., Eriksson, K., et al. 2008, A\&A, 486, 951

King, I. 1962, AJ, 67, 471

Lépine, J. R. D., Cruz, P., Scarano, S., Jr., et al. 2011, MNRAS, 417, 698

Li, Z., Mao, C., Chen, L., \& Zhang, Q. 2012, ApJ, 761, L22

Magrini, L., Sestito, P., Randich, S., \& Galli, D. 2009, A\&A, 494, 95

Magrini, L., Randich, S., Friel, E., et al. 2013, A\&A, 558, A38

Magrini, L., Randich, S., Romano, D., et al. 2014, A\&A, in press

Malkin, Z. 2013, IAU Symp., 289, 406

McSwain, M. V., \& Gies, D. R. 2005, ApJS, 161, 118

Mermilliod, J.-C., \& Mayor, M. 1989, A\&A, 219, 125

Mermilliod, J.-C., \& Mayor, M. 1990, A\&A, 237, 61

Mermilliod, J.-C., Claria, J. J., Andersen, J., \& Mayor, M. 1997, A\&A, 324, 91

Mermilliod, J.-C., Mathieu, R. D., Latham, D. W., \& Mayor, M. 1998, A\&A, 339,423

Miglio, A., Brogaard, K., Stello, D., et al. 2012, MNRAS, 419, 2077

Milone, A. P., Bedin, L. R., Piotto, G., \& Anderson, J. 2009, A\&A, 497, 755

Milone, A. P., Piotto, G., Bedin, L. R., et al. 2012, A\&A, 540, A16

Pancino, E., Carrera, R., Rossetti, E., \& Gallart, C. 2010, A\&A, 511, A56

Pasquini, L., Avila, G., Blecha, A., et al. 2002, The Messenger, 110, 1

Pietrinferni, A., Cassisi, S., Salaris, M., \& Castelli, F. 2004, ApJ, 612, 168

Platais, I., Melo, C., Fulbright, J. P., et al. 2008, MNRAS, 391, 1482

Platais, I., Melo, C., Quinn, S. N., et al. 2012, ApJ, 751, L8

Randich, S., \& Gilmore, G. 2013, The Messenger, in press

Robin, A. C., Reylé, C., Derrière, S., \& Picaud, S. 2003, A\&A, 409, 523

Seleznev, A. F., Carraro, G., Costa, E., \& Loktin, A. V. 2010, New Astron., 15, 61

Skrutskie, M. F., Cutri, R. M., Stiening, R., et al. 2006, AJ, 131, 1163

Tosi, M., Bragaglia, A., \& Cignoni, M. 2007, MNRAS, 378, 730

Vallée, J. P. 2005, AJ, 130, 569

VandenBerg, D. A., Bergbusch, P. A., \& Dowler, P. D. 2006, ApJS, 162, 375

Yang, W., Meng, X., Bi, S., et al. 2011, ApJ, 731, L37

Yong, D., Carney, B. W., \& Friel, E. D. 2012, AJ, 144, 95 